Japan. J. Math.

Vol. 24, No. 2, 1998

\title{
Some oscillation results for linear differential equations in the complex plane
}

\author{
By Yik-Man Chiang and Ilpo Laine \\ (Received February 19, 1996) \\ (Revised March 13, 1998) \\ (from Nagoya Mathematical Journal)
}

\section{Introduction}

Since the beginning of the last decade, a substantial number of research articles have been written to describe the frequency of zeros of solutions of linear differential equations of type,

$$
f^{\prime \prime}+A(z) f=0
$$

where $A(z)$ is a transcendental entire function, see [1] for the starting point of these investigations. The exponent of convergence $\lambda(f)$ of the zero-sequence of $f$ is the standard device to measure the frequency of the zeros. Now, let $f_{1}, f_{2}$ be two linearly independent solutions of (1.1). The major conjecture, still remaining open, is that whenever the order $\sigma(A)$ of $A$ is finite and not an integer, then $\max \left(\lambda\left(f_{1}\right), \lambda\left(f_{2}\right)\right)=$ $+\infty$. Most of the research work dealing with (1.1) during the last decade has been directed towards proving this conjecture, at least partially. This explains why several papers have been written to describe equations of type (1.1) such that all solutions admit plenty of zeros, say in the sense that $\lambda(f)=\infty$. A typical such result is the following theorem (see [14], Theorem 5.7).

Theorem A. All non-trivial solutions $f$ to

$$
f^{\prime \prime}+e^{P(z)} f=0
$$

where $P(z)$ is a non-constant polynomial, satisfy $\lambda(f)=+\infty$.

A related result, see [14], Theorem 5.17, which may be understood as a kind of perturbation theorem, is

Theorem B. Let $P$ be a polynomial of degree $\operatorname{deg} P=\lambda>0$, and $Q$ be an entire function of order $\sigma(Q)<\lambda$. Suppose that

Supported in part by the Finnish Academy grants 1021097 and 2592, by the University of Joensuu and by the Hong Kong University of Science and Technology grant DAG92/93.SC22. 


$$
f^{\prime \prime}+\left(e^{P}+Q\right) f=0
$$

admits a non-trivial solution $f$ such that $\lambda(f)<\lambda$. Then $f$ has no zeros, $Q$ is a polynomial and

$$
Q=-\frac{1}{16}\left(P^{\prime}\right)^{2}+\frac{1}{4} P^{\prime \prime}
$$

Moreover, (1.3) admits in this special case two linearly independent zero-free solutions.

These results, as well as a number of related theorems, raise the natural possibility that the zero deficiency of $A(z)$, or perhaps more generally its defect sum, may have some consequences for the zero frequency of $f$. The first results of this type were obtained by Y. Chiang in his London thesis [7], partially improved by Y. Chiang [8].

The aim of this article is twofold. We first give, in Section 2, one more deficiency type result in the basic second order case (1.1). In fact, this result may be understood as an improvement of Theorem B. Secondly, we give, in Section 4, two deficiency type results for the higher order case

$$
f^{(k)}+A(z) f=0 .
$$

This will be accomplished by proving, in Section 6, a modification of the following theorem due to S. Bank and J. Langley (see [6], Theorem 4).

Theorem C. Suppose that $k \geq 2$, and that $A(z)$ is a transcendental entire function of finite order $\sigma$ with the following property: there exists a set $H \subset \mathbb{R}$ of measure zero such that for each real $\theta \notin H$ either

(i) $r^{-N} \mid A\left(r e^{i \theta} \mid \rightarrow+\infty\right.$ as $r \rightarrow \infty$ for each $N>0$,

or

$$
\text { or }
$$$$
\text { (ii) } \int_{0}^{\infty} r^{k-1}\left|A\left(r e^{i \theta}\right)\right| d r<+\infty
$$

(iii) there exist constants $K>0, b>0$ and $n \geq 0$ such that $n+k<k \sigma$ and

$$
\left|A\left(r e^{i \theta}\right)\right| \leq K r^{n} \quad \text { for all } r \geq b .
$$

Then the equation

$$
f^{(k)}+A(z) f=0
$$

cannot have a fundamental set of $k$ linearly independent solutions $f_{1}, \ldots, f_{k}$ each with finite exponent of convergence of zeros.

For an application of this theorem, see [2], Corollary 1: 
Corollary D. Let $n \geq 1$, and let $P_{1}, \ldots, P_{n}$ be non-constant polynomials whose degrees are $d_{1}, \ldots, d_{n}$ respectively, and suppose that for $i \neq j$,

$$
\operatorname{deg}\left(P_{i}-P_{j}\right)=\max \left\{d_{i}, d_{j}\right\}
$$

Set

$$
A(z)=\sum_{j=1}^{n} B_{j}(z) e^{P_{j}(z)}
$$

where, for each $j, B_{j}(z)$ is an entire function, not identically zero, of order strictly less than $d_{j}$. Then if $f_{1}$ and $f_{2}$ are linearly independent solutions of

$$
y^{\prime \prime}+A y=0
$$

we have $\max \left\{\lambda\left(f_{1}\right), \lambda\left(f_{2}\right)\right\}=+\infty$.

The same conclusion holds for the equation

$$
y^{\prime \prime}+(A+P) y=0,
$$

where $P(z)$ is a polynomial whose degree $m$ satisfies $(m+2) / 2<\sigma(A)=\max \left\{d_{j}\right\}$.

In what follows, we assume that the reader is familiar with the standard notations and results of the Nevanlinna theory (see e.g. [12] and [14]).

\section{The second order case}

The following theorem will be proved similarly as to Theorem B, see e.g. [3], Theorem 3.3.

THEOREM 1. Let $A$ be an entire function of finite order $\lambda$ such that $\bar{N}\left(r, \frac{1}{A}\right)=$ $S(r, A)$, and let $Q$ be an entire function such that $T(r, Q)=S(r, A)$. Suppose that

$$
f^{\prime \prime}+(A(z)+Q(z)) f=0
$$

admits a non-trivial solution $f$ such that $N\left(r, \frac{1}{f}\right)=S(r, A)$. Then $A(z)$ has no zeros, and so there is a polynomial $P(z)$ of degree $\lambda$ such that $A(z)=\exp P(z)$. Moreover, $f$ has no zeros, $Q$ is a polynomial,

$$
Q=-\frac{1}{16}\left(P^{\prime}\right)^{2}+\frac{1}{4} P^{\prime \prime}
$$

and (2.1) admits two linearly independent zero-free solutions.

Proof. We write $f$ in the form $f=H e^{g}$, where $g$ is transcendental entire and $H$ is the canonical product formed with the zeros of $f$. By our assumptions, $\bar{N}\left(r, \frac{1}{H}\right)=N\left(r, \frac{1}{H}\right)=S(r, A)$. Substituting $f$ into (2.1) we obtain

$$
-\left(g^{\prime}\right)^{2}=g^{\prime \prime}+2 \frac{H^{\prime}}{H} g^{\prime}+\frac{H^{\prime \prime}}{H}+A+Q .
$$


Now, we easily see that the canonical product $H$ is of finite order $\leq \lambda$, hence $m\left(r, \frac{H^{\prime}}{H}\right)=O(\log r)$. By the standard Clunie argument (see [14], Lemma 2.4.2), (2.2) results in $\sigma\left(g^{\prime}\right) \leq \lambda$. The reversed inequality follows from (2.2) by elementary arguments, hence $\sigma(g)=\sigma\left(g^{\prime}\right)=\lambda$. Similarly as in [3], we now obtain

$$
\begin{aligned}
g^{\prime}\left(2 g^{\prime \prime}-\frac{A^{\prime}}{A} g^{\prime}\right)= & -g^{\prime \prime \prime}+\left(\frac{A^{\prime}}{A}-2 \frac{H^{\prime}}{H}\right) g^{\prime} \\
& +\left\{2 \frac{A^{\prime}}{A} \frac{H^{\prime}}{H}-2\left(\frac{H^{\prime}}{H}\right)^{\prime}\right\} g^{\prime}+\frac{A^{\prime}}{A} \frac{H^{\prime \prime}}{H}-\left(\frac{H^{\prime \prime}}{H}\right)^{\prime}+\frac{A^{\prime}}{A} Q-Q^{\prime} .
\end{aligned}
$$

Now,

$$
T\left(r, \frac{A^{\prime}}{A}\right)=m\left(r, \frac{A^{\prime}}{A}\right)+\bar{N}\left(r, \frac{1}{A}\right)=S(r, A) .
$$

By the Clunie argument once more, we see that

$$
g^{\prime \prime}-\frac{1}{2} \frac{A^{\prime}}{A} g^{\prime}=B(z)
$$

where $m(r, B)=S(r, A)$. Moreover, it is clear that all poles of $B$ are simple and among the zeros of $A$. Hence

$$
T(r, B) \leq m(r, B)+\bar{N}\left(r, \frac{1}{A}\right)=S(r, A) .
$$

Now, the same reasoning as applied in [3] results in the following equality:

$$
\begin{aligned}
\left\{2\left(\frac{H^{\prime}}{H}\right)^{\prime}+\right. & \left.\frac{1}{2}\left(\frac{A^{\prime}}{A}\right)^{\prime}-\frac{A^{\prime}}{A} \frac{H^{\prime}}{H}-\frac{1}{4}\left(\frac{A^{\prime}}{A}\right)^{2}+2 B\right\} g^{\prime} \\
& +\left(\frac{H^{\prime \prime}}{H}\right)^{\prime}+B^{\prime}+Q^{\prime}+2 B \frac{H^{\prime}}{H}-\frac{1}{2} \frac{A^{\prime}}{A} B-\frac{A^{\prime}}{A} \frac{H^{\prime \prime}}{H}-\frac{A^{\prime}}{A} Q=0 .
\end{aligned}
$$

Therefore, $T\left(r, g^{\prime}\right)=S(r, A)$, by elementary Nevanlinna theory estimates, and $T(r, A)=S(r, A)$ by $(2.2)$. This is a contradiction, unless we have

$$
2\left(\frac{H^{\prime}}{H}\right)^{\prime}+\frac{1}{2}\left(\frac{A^{\prime}}{A}\right)^{\prime}-\frac{A^{\prime}}{A} \frac{H^{\prime}}{H}-\frac{1}{4}\left(\frac{A^{\prime}}{A}\right)^{2}+2 B=0
$$

and

$$
\left(\frac{H^{\prime \prime}}{H}\right)+B^{\prime}+Q^{\prime}+2 B \frac{H^{\prime}}{H}-\frac{1}{2} \frac{A^{\prime}}{A} B-\frac{A^{\prime}}{A} \frac{H^{\prime \prime}}{H}-\frac{A^{\prime}}{A} Q=0 .
$$

Now, a zero $z_{0}$ of $H$ has to be a zero of $A$, say of order $\beta>0$, while the zero of $H$ at $z_{0}$ is simple. An elementary Laurent expansion consideration at $z_{0}$, applied 
to $(2.3)$ yields $\beta^{2}+6 \beta+8=0$, a contradiction for $\beta>0$. Hence $H$ has no zeros and we may assume $H=1$. Therefore (2.2) and (2.3) reduce into

$$
B=-\frac{1}{4}\left(\frac{A^{\prime}}{A}\right)^{\prime}+\frac{1}{8}\left(\frac{A^{\prime}}{A}\right)^{2}
$$

and

$$
Q^{\prime}-\frac{A^{\prime}}{A} Q+B^{\prime}-\frac{1}{2} \frac{A^{\prime}}{A} B=0
$$

Since all poles of $B$ must be simple, (2.5) reveals at once that $A$ cannot have zeros. Writing $A(z)=e^{P(z)}$ where $P$ is a polynomial we obtain

$$
\left\{\begin{array}{l}
B=-\frac{1}{4} P^{\prime \prime}+\frac{1}{8}\left(P^{\prime}\right)^{2} \\
Q^{\prime}-P^{\prime} Q+B^{\prime \prime}-\frac{1}{2} P^{\prime} B=0
\end{array}\right.
$$

from (2.5) and (2.6). The assertion now follows exactly as in the proof of [3], Theorem 3.3 .

\section{Discussion on previous results}

The main application of Theorem $\mathrm{C}$ is Corollary $\mathrm{D}$ where the coefficient $A(z)$ in (1.7) has the form (1.6) whose behaviour in $\mathbb{C}$ and especially its radial growth properties satisfy (i), (ii) and (iii) of Theorem $\mathrm{C}$ except perhaps for a subset of $[0,2 \pi)$ of measure zero.

The above results lead one to consider, quite naturally, other classes of functions $A(z)$ as coefficients which have similar growth regularities as those of (1.6). One such class of functions was considered in [8] (see also [7]), where the functions have maximal sum of deficiencies, i.e., $\sum_{a \in \mathbb{C}} \delta(a, A)=1$ where $\delta(a, A)$ or simply $\delta(a)$ denotes the Nevanlinna deficiency of $A$ at $a$; we refer to [12] for details. Two results were proved in [8]:

THEOREM E. Let $A(z)$ be a transcendental entire function of finite order $\sigma(A)$, satisfying $\delta(0, A)=1$.

(a) Suppose $f_{1}$ and $f_{2}$ are linearly independent solutions of

$$
f^{\prime \prime}+A(z) f=0
$$

Then $\max \left\{\lambda\left(f_{1}\right), \lambda\left(f_{2}\right)\right\}=\infty$.

(b) Suppose further that $P(z)$ is a non-constant polynomial of degree $n$ and

$$
n+2<2 \sigma(A) \text {. }
$$


Then the same conclusion as that of (a) holds for any two linearly independent solutions of

$$
f^{\prime \prime}+(A(z)+P(z)) f=0
$$

Theorem F. Let $A(z)$ be a transcendental entire function of finite order $\sigma(A)$ and $\sum \delta(a, A)=1$ and $P(z)$ be a polynomial with degree $n \geq 0$ and $n+2<$ $2 \sigma(A)$. Then the equation

$$
f^{\prime \prime}+(A(z)+P(z)) f=0
$$

cannot have a fundamental set of solutions each with finite exponent of convergence of zeros.

We note that the function $e^{P}$, where $P$ is a polynomial, satisfies $\sum_{a \in \mathbb{C}} \delta(a)=$ $\delta(0)=1$ trivially, and the example

$$
f^{\prime \prime}-\frac{1}{4}\left(e^{-2 z^{n}}+n^{2} z^{2 n-2}+2 n(n-1) z^{n-2}\right) f=0
$$

as discussed in [1] and [2; p. 20], admits two linearly independent solutions which are both zero free.

The inequality (3.1) and the same one in Theorem $\mathrm{F}$ exclude the possibility that $P(z)$ is identically a constant and the order of $A(z)$ equals one. For suppose $P \equiv 0$ and thus $\operatorname{deg} P=0$. Hence (3.1) implies $\sigma(A)>1$ excluding the range $\sigma(A) \leq 1$. Hence part (a) of Theorem $\mathrm{E}$ is not included in (b). We note that Theorem $\mathrm{F}$ also does not include the case (a) of Theorem $\mathrm{E}$ for the same reason. However, Theorem $\mathrm{F}$ does include Theorem $\mathrm{E}$ (b) but their proofs are different. The example $f^{\prime \prime}+\left(e^{z}-K\right) f=0$ considered in [2, Theorem 2] which corresponds to (3.2) when $n=1$ shows that it can possess two linearly independent solutions each of which has a finite exponent of convergence of zeros as soon as $K=n^{2} / 16$, where $n$ is a positive odd integer.

The above examples show that both (4.1) and (4.2) of Theorems E and F respectively are sharp in these two aspects.

In fact, the growth properties of functions with $\delta(0, A)=1$ or $\sum_{a} \delta(a, A)=1$ differ, generally, from the function $A(z)$ in Theorem $\mathrm{C}$. Instead of the radial growth estimates (i), (ii) and (iii) of Theorem $\mathrm{C}$, we now have functions which have growth estimates in different chains of connected regions. In fact, there exists a sequence of annuli with both of its radii tending to infinity and the intersection of any two consecutive annuli is non-empty (in fact the intersection has an area tending to infinity [10]). Each of the annulus is divided into a same number of equal sectorial regions (the number depends on the order of the function), and each chain is the union of these sectorial regions, one from each annulus, and the union is connected in a certain way so that each chain stretches to infinity. An estimate of $A(z)$ on 
different chains can be extracted from [10]. An account of the estimate mentioned will be given below, as far as their applications require it. (see Lemmas 10, 11 and 12 in $\S 9)$.

The main purpose in the next section is to generalize the above Theorems to higher order equations.

\section{Notations and higher order results}

We obtain higher order analogues of Theorems E and F.

Theorem 2. Let $k$ be a positive integer $\geq 2$ and $A(z)$ be a transcendental entire function of order $\sigma(A)<\infty$, satisfying $\delta(0, A)=1$, and $P(z)$ be a polynomial of degree $n$. Suppose that $f(z)$ is a non-trivial solution of either

$$
f^{(k)}+A(z) f=0
$$

or

$$
f^{(k)}+(A(z)+P(z)) f=0,
$$

provided $n \geq 0$ and $n+k<k \sigma(A)$.

Then, in both cases, the corresponding equation cannot have a fundamental set of solutions each with finite exponent of convergence of zeros.

THEOREM 3. Let $k \geq 2$ be a positive integer and let $A(z)$ be a transcendental entire function of finite order $\sigma(A)$ and $\sum \delta(a, A)=1$ and $P(z)$ be a polynomial with degree $n \geq 0$ and $n+k<k \sigma(A)$. Then the equation

$$
f^{(k)}+(A(z)+P(z)) f=0
$$

cannot have a fundamental set of solutions each with finite exponent of convergence of zeros.

Remark. Observe that $k+n<k \sigma(A)$ implies $\sigma(A)>1$ when $n=0$, and hence the case for (4.1) is not included in that of $(4.2)$ when $\sigma(A)=1$. The same reason applies to (4.3) (that is $\sigma(A)>1$ is assumed in Theorem 3). See the paragraph after Theorem F.

We shall give examples to the theorems to indicate their sharpness in $\S 10$.

Theorems 2 and 3 follow from a more general result to be stated below. We mention that Theorems 2 and 3 already encompass Theorems $\mathrm{E}$ and F. However, the method of proofs are different. But before we can state our result, we need some notations and definitions.

Let $I=[1, \infty)$ and $F \subset I$. Then

$$
m(F(r)):=\int_{t \in F(r)} d t, \quad \operatorname{lm}(F(r)):=\int_{t \in F(r)} \frac{1}{t} d t
$$


are the linear and logarithmic measures of $F(r)=F \cap[1, r]$, respectively.

We also define

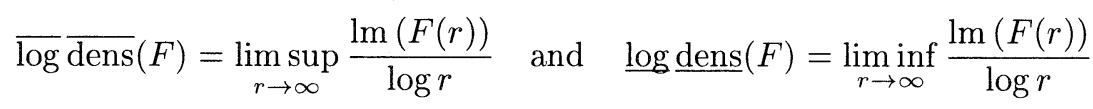

to be the upper and lower logarithmic densities of $F$. Note that

$$
\overline{\log } \overline{\operatorname{dens}}(I)=1=\underline{\log \underline{\operatorname{dens}}}(I) \quad \text { and } \quad \overline{\log } \overline{\operatorname{dens}}(F)=1-\underline{\log \underline{\operatorname{dens}}}(I \backslash F) .
$$

Let $c$ and $d$ be positive numbers, and $p$ be a fixed positive integer. We define a sequence of annuli $\Gamma_{j}=\left\{z: r_{1, j} \leq|z| \leq r_{2, j}\right\}$ where $\log r_{1, j}=c j, \log r_{2, j}=c j+d$, $d>c$ so that $r_{2, j}-r_{1, j} \rightarrow+\infty$ as $j \rightarrow+\infty$, and $r_{2, j}-r_{1, j}>A$ where $A$ is a constant depending only on $p$. Note also that $r_{2, j}-r_{1, j+1} \rightarrow \infty$. Hence $\Gamma_{j}$ and $\Gamma_{j+1}$ overlap each other for all $j$ sufficiently large.

Let $p \geq 2$ be a fixed integer. For each $\varepsilon>0$ and $j$, we define $p$ angles so that $\theta_{1, j}<\theta_{2, j}<\cdots<\theta_{p, j}<\theta_{1, j}+2 \pi$ and for each $l$ in $\{1, \ldots, p\}$, we have

$$
4 \varepsilon<\left|\theta_{l+1, j}-\theta_{l, j}\right|
$$

and

$$
\left|\theta_{l, j+1}-\theta_{l, j}\right|<\arcsin (\varepsilon)
$$

for all $j$ sufficiently large, where we set $\theta_{p+1}=\theta_{1}$. We shall write $\theta_{l}, l=1, \ldots, p$ instead of the $\theta_{l, j}$ when the dependence of $j$ is clear from the text, and we shall adopt this principle to suppress the subscripts $j$ in the following definitions. The union of the sets

$$
P_{l, \varepsilon}:=\left\{\theta: \theta_{l}-\varepsilon \leq \theta \leq \theta_{l}+\varepsilon\right\}
$$

and

$$
Q_{l, \varepsilon}:=\left\{\theta: \theta_{l}+\varepsilon \leq \theta \leq \theta_{l+1}-\varepsilon\right\}
$$

for $l=1, \ldots, p$, is $[0,2 \pi]$; that is $\bigcup_{l}\left\{Q_{l, \varepsilon} \cup P_{l, \varepsilon}\right\}=[0,2 \pi]$.

We also define for a fixed $\varepsilon>0$ the following sets:

$$
Q_{l, \varepsilon}\left(r_{1, j}, r_{2, j}\right):=\left\{r e^{i \theta}: \theta \in Q_{l, \varepsilon} \text { and } r \in\left[r_{1, j}, r_{2, j}\right]\right\}
$$

such that $\bigcup_{j} Q_{l, \varepsilon}\left(r_{1, j}, r_{2, j}\right)$ is a connected set for each $l=1, \ldots, p$ since $d>c$. Similarly, we define

$$
P_{l, \varepsilon}\left(r_{1, j}, r_{2, j}\right):=\left\{r e^{i \theta}: \theta \in P_{l, \varepsilon} \text { and } r \in\left[r_{1, j}, r_{2, j}\right]\right\} \text {. }
$$

Let $H \subset \mathbb{C}$ be a disjoint, locally finite union of discs of different sizes and $H_{P}$ the projection of $H$ onto the positive real axis by clockwise rotation about the origin. 
We define in addition

$$
Q_{l, \varepsilon, H}\left(r_{1, j}, r_{2, j}\right):=Q_{l, \varepsilon}\left(r_{1, j}, r_{2, j}\right) \backslash H .
$$

We are ready to state our result.

THEOREM 4. Let $p \geq 2$ be a positive integer. Suppose that for any $\varepsilon, \Delta$ such that $0<\Delta<\varepsilon<1 / 3$, there exist sequences of sectorial domains $Q_{l, \varepsilon, H}\left(r_{1, j}, r_{2, j}\right)$ for $l=1, \ldots, p$, as defined in Section 4 , such that $\overline{\log } \overline{\operatorname{dens}}\left(H_{P}\right)=0$ and for all $j$ sufficiently large we have the angular measure of the exceptional set in $\Gamma_{j}=\{z=$ $\left.r e^{i \theta}: r_{1, j} \leq r \leq r_{2, j}\right\}$ to be less then $\Delta$. Let $A(z)$ be a transcendental entire function of finite order $\sigma(A)$ satisfying, for each $l \in\{1, \ldots, p\}$, either

(i) $|A(z)| / r^{m} \rightarrow \infty$ for each $m>0$ uniformly in $\bigcup^{\infty} Q_{l, \varepsilon, H}\left(r_{1, j}, r_{2, j}\right)$,

or

(ii) $r^{m}|A(z)| \rightarrow 0$ for each $m>0$ uniformly in $\bigcup^{\infty} Q_{l, \varepsilon, H}\left(r_{1, j}, r_{2, j}\right)$,

or

(iii) there exist $K, n>0$ such that $n+k<k \sigma(A)$ and that

$$
A(z) \leq K r^{n}
$$

uniformly in $\bigcup^{\infty} Q_{l, \varepsilon, H}\left(r_{1, j}, r_{2, j}\right)$.

Then the equation

$$
f^{(k)}+A(z) f=0
$$

cannot have a fundamental set of solutions $\left\{f_{1}, \ldots, f_{k}\right\}$ such that each $f_{i}$ has a finite exponent of convergence of zeros.

We now discuss whether Theorem 4 still hold if we allow (4.12) to have some intermediate terms. Let $A(z)=e^{z}$, then the sectorial regions $\bigcup^{\infty} Q_{l, \varepsilon}\left(r_{1, j}, r_{2, j}\right)$, $l=1,2$ become infinite sectors, and the coefficient satisfies the growth conditions of (i) and (ii) of Theorem 4. However it follows from Theorem 1 in [9] (see $\S 10$ ) that equation (4.12) $(k=3)$ with an intermediate term involving $f^{\prime}$ admits a fundamental set of solutions $f$ each with $\lambda(f)<+\infty$. Hence we generally cannot allow any intermediate coefficients in (4.1) without imposing further assumptions. Hence Theorem 4 is sharp in this sense.

Although we shall base part of our argument on that of [6], the remaining part does not seem to carry over from [6] to our problem. This is because the methods in [6] are mostly based on radial growth estimates. Hence new techniques (estimates in annuli) are required and this will be established from Lemmas 3 to 9 in $\S 5$. In particular Lemmas 8 and 9 are of independent interest. 


\section{Lemmas required to prove Theorem 4}

Although our proof of Theorem 4 goes in the same spirit as those of Theorems $\mathrm{E}$ and $\mathrm{F}$, several parts of it differ substantially from those of the corresponding Theorems $\mathrm{E}$ and $\mathrm{F}$. In the second order case, we can make use of a special non-linear differential equation $4 A E^{2}=\left(E^{\prime}\right)^{2}-c^{2}-2 E E^{\prime \prime}$ and the third-order linear differential equation $E^{\prime \prime \prime}+4 A E^{\prime}+2 A^{\prime} E=0$ where $E$ is the product of any two linearly independent solutions to $(1,1)$ and the constant $c$ is their Wronskian. Corresponding equations for higher order cases $k \geq 3$, are not known to exist. However, useful techniques were developed in [6; Theorem 4] upon which part of our argument is based.

Next, we note that any non-trivial solution of the equation (1.5) can be written in the following integrated form:

$$
\begin{aligned}
y(z)=c_{0}+c_{1}\left(z-z_{0}\right)+\cdots+c_{k-1}\left(z-z_{0}\right)^{k-1} \\
-\frac{1}{(k-1) !} \int_{z_{0}}^{z}(z-s)^{k-1} A(s) y(s) d s
\end{aligned}
$$

where $c_{0}=y\left(z_{0}\right), c_{1}=y^{\prime}\left(z_{0}\right), \ldots, c_{k-1}=y^{(k-1)}\left(z_{0}\right) /(k-1)$ !.

We shall also make use of the important fact that for any non-trivial solution $f=\pi e^{h}$ of $(1.5), h(z)$ is of finite order, provided $\pi(z)$ is of finite order. This follows by a standard Clunie type argument (see e.g. [3], p. 11).

In addition, we require the following lemmas.

Lemma 1. Let $\xi \in[0,1 / 6)$ be a real number, and $g(r)$ and $h(r)$ are both monotone increasing functions defined on $(0,+\infty)$. Suppose that $g(r) \leq h(r)$ for all $r \in(0,+\infty)$ except perhaps on an exceptional set $E$ of $\overline{\log } \overline{\operatorname{dens}}(E) \leq \xi$. Then for every $0<\varepsilon<\xi<1 / 6$ sufficiently small, there exists a $r_{1}=r_{1}(\varepsilon)>0$ such that $g(r) \leq h\left(r^{1+2 \xi+\varepsilon}\right)$ for all $r>r_{1}$.

Proof. Suppose $\overline{\log } \overline{\operatorname{dens}}(E) \leq \xi$. Let $E(r)=E \cap[1, r)$. For every $\varepsilon>0$, there exists an $r_{1}=r_{1}(\varepsilon)$ such that

$$
\int_{t \in E(r)} \frac{d t}{t}<(\xi+\varepsilon) \log r<\frac{3}{2} \xi \log r
$$

for all $r>r_{1}$.

We claim that for each $r>0$ not in $E$, there exists a $t \in\left(r, r^{\prime}\right)$ but $t \notin E$, where $r^{\prime}=r^{1+2 \xi+\varepsilon}$. For suppose this is not the case, then $\left(r, r^{\prime}\right)$ is a proper subset of $E$. But then

$$
\frac{3}{2} \xi \log r^{\prime}>\int_{t \in E\left(r^{\prime}\right)} \frac{d t}{t} \geq \int_{t \in\left(r, r^{\prime}\right)} \frac{d t}{t}=\log r^{\prime}-\log r .
$$


It follows from the above inequality and the choice of $r^{\prime}$ that

$$
\left(1-\frac{3}{2} \xi\right)(1+2 \xi+\varepsilon)<1
$$

But $\left(1-\frac{3}{2} \xi\right)(1+2 \xi)>1$ for $\xi<1 / 6$. By choosing $\varepsilon>0$ sufficiently small while taking $r_{1}$ sufficiently large, we have $\left(1-\frac{3}{2} \xi\right)(1+2 \xi+\varepsilon)>1$ when $\xi<1 / 6$. This is a contradiction to the hypothesis on $\xi$. Thus for every $r>r_{1}$ there must exist a $t \in\left(r, r^{1+2 \xi+\varepsilon}\right)$ and $t \notin E$. Hence, for all $r>r_{1}$, we have

$$
g(r) \leq g(t) \leq h(t) \leq h\left(r^{1+2 \xi+\varepsilon}\right) .
$$

Lemma 2 ([11], Lemma 1). Let $h(z)$ be meromorphic in $\mathbb{C}$ and of finite order $\sigma$. Then given $\xi>0$ and $0<\delta<1 / 2$, there exist a constant $K(\sigma, \xi)$ and a set of positive real numbers $G$ of lower logarithmic density at least $1-\xi>0$ such that if $0 \leq \theta_{2}-\theta_{1} \leq \delta<1 / 2$ and $r \in G$, then

$$
r \int_{\theta_{1}}^{\theta_{2}}\left|\frac{h^{\prime}}{h}\left(r e^{i \theta}\right)\right| d \theta<K(\rho, \xi)\left(\delta \log \frac{1}{\delta}\right) T(r, h) .
$$

Lemma 3 ([13], Satz 1). Let $p_{j}(x), j=1, \ldots, k$, and $f(x)$ be complex-valued functions in $[a, b)$. Suppose $P_{j}(x), j=1, \ldots, k$, and $F(x)$ are continuous, nonnegative functions defined on $[a, b)$ such that $\left|p_{j}(x)\right| \leq P_{j}(x)$ and $|f(x)| \leq F(x)$. Let $v(x)$ satisfy the differential equation

$$
v^{(k)}-\sum_{j=1}^{k} p_{j}(x) v^{(k-j)}=f(x),
$$

and $V(x)$ satisfy the differential equation

$$
V^{(k)}-\sum_{j=1}^{k} P_{j}(x) V^{(k-j)}=F(x) .
$$

Moreover, we assume that

$$
\left|v^{(m)}(a)\right| \leq V^{(m)}(a) \quad \text { for } m=0, \ldots, k-1 .
$$

Then

$$
\left|v^{(m)}(x)\right| \leq V^{(m)}(x) \quad \text { for } m=0, \ldots, k-1
$$

holds for $x \in[a, b)$ 
REMARK. We note that Herold's original result is more general. However, the present version is sufficient for our applications.

By examining the proof of [3, Lemma 3] we see that the following lemma is valid. Only the conclusion when $m=0$ was shown in [4].

Lemma 4. Suppose that $A(z)$ is analytic in a sector $S$ containing the ray $z=r e^{i \theta}$ and that, for some non-negative $K$ and some $r_{0}>0$, we have

$$
\left|A\left(r e^{i \theta}\right)\right| \leq K r^{n}
$$

for all $r \geq r_{0}$. If $f(z)$ is a non-trivial solution of (1.5), then there exist positive constants $M$ and $N$ such that

$$
\left|f^{(m)}\left(r e^{i \theta}\right)\right| \leq M \exp (\eta(r)), \quad m=0, \ldots, k-1,
$$

holds for all $r \geq r_{0}$, where $\eta(r):=N r^{(n+k) / k}$.

LEMma 5. Let $f(z)$ be an entire function, and

$$
F(t):=f\left(r e^{i \Theta}\right), \quad \Theta(t):=a-t(a-b),
$$

for $0 \leq t \leq 1$ and $a, b \in[0,2 \pi),|a-b| \geq \Delta>0$ for a fixed but suitable $\Delta, 0<\Delta<$ 1. Let $k$ be any positive integer then there exist positive constants $D, D^{\prime}$ and $r_{1}$ (depending only on $k$ and not on $f$ ) such that for $m=1, \ldots, k-1,0 \leq t \leq 1$ and for $r \geq r_{1}>r_{0}$ where $r_{0}$ is defined in Lemma 4, the following estimates hold:

(a) $\left|F^{(m)}(t)\right| \leq(2 \pi)^{m} r^{m} D \sum_{l=1}^{m}\left|f^{(l)}\left(r e^{i \Theta}\right)\right|$

and

(b) $\left|f^{(m)}\left(r e^{i \Theta}\right)\right| \leq \frac{(2 \pi)^{m} D^{\prime}}{(\Delta r)^{m}} \sum_{l=1}^{m}\left|F^{(l)}(t)\right|$.

Proof. (a) It is easily established, by induction, on differentiating $F$ repeatedly $m$ times with respect to $t$, that

$$
F^{(m)}(t)=\nu^{m} \sum_{j=1}^{m} a_{j}\left(r e^{i \Theta}\right)^{j} f^{(j)}\left(r e^{i \Theta}\right)
$$

where $\nu=a-b$, and $a_{i}, i=1, \ldots, m$ are constants and $\left|a_{m}\right|=\left|a_{1}\right|=1$. Let $D=\max \left(\left|a_{i}\right|\right)$. Then (5.3) follows by induction.

(b) Similarly, by making use of the identity above repeatedly, we have

$$
f^{(m)}\left(r e^{i \Theta}\right)=\frac{1}{\left(i \nu r e^{i \Theta}\right)^{m}}\left(b_{m} F^{(m)}(t)+b_{m-1} \nu F^{(m-1)}(t)+\cdots+b_{1} \nu^{m-1} F^{\prime}(t)\right),
$$


where $b_{i}, i=1, \ldots, m$ are constants and $\left|b_{m}\right|=\left|b_{1}\right|=1$. Now (5.4) follows by choosing $D^{\prime}=\max \left(\left|b_{i}\right|\right)$, and noting that $\left|\nu^{-m}\right| \leq \Delta^{-m}$.

Lemma 6. Let $g(r, t):=\exp (\eta(r)(t+\beta))$ be defined for $r>r_{0}$ and for $t \in[0,1]$, where $\eta(r)=N r^{\frac{n+k}{k}}$ is as defined in Lemma 4 , and $\beta \geq 1$ is a constant. Let $k$ be a positive integer, then there exist a positive number $A_{1}$ (depending only on $k$ ) and $r_{2}>0$ such that $r_{2}>r_{1}>r_{0}$ and for all $r>r_{2}$,

$$
\left(\frac{\eta(r)}{r}\right)^{m} g(r, t) \leq g_{r}^{(m)}(r, t) \leq A_{1}(2 \beta)^{m}\left(\frac{\eta(r)}{r}\right)^{m} g(r, t), \quad m=1, \ldots, k,
$$

where the subscript $r$ in (5.6) indicates the partial differentiation is taken with respect to $r$, while $t$ is being kept fixed. Here $r_{1}$ is the same in Lemma 5.

Proof. Differentiating $g$ with respect to $r$ once gives

$$
g_{r}^{\prime}(r, t)=N(t+\beta)\left(1+\frac{n}{k}\right) r^{\frac{n}{k}} g(r, t)
$$

A simple induction then implies (see also [12], Lemma 3.5)

$$
g_{r}^{(m)}(r, t)=\left(N^{m}(t+\beta)^{m}\left(1+\frac{n}{k}\right)^{m} r^{m \frac{n}{k}}+O\left(r^{(m-1) \frac{n}{k}-1}\right)\right) g(r, t)
$$

for $m=1, \ldots, k-1$.

We deduce, when $r \geq r_{2}$ for some suitably chosen $r_{2}>r_{1}$,

$$
\frac{g_{r}^{(m)}(r, t)}{g(r, t)} \leq N^{m}(2 \beta)^{m} 2^{m} r^{m \frac{n}{k}}=A_{1}(2 \beta)^{m}\left(\frac{\eta(r)}{r}\right)^{m},
$$

and

$$
\frac{g_{r}^{(m)}(r, t)}{g(r, t)} \geq\left(N r^{\frac{n}{k}}\right)^{m}=\left(\frac{\eta(r)}{r}\right)^{m}
$$

and this gives the required estimates.

LEMma 7. Let $F(t)$ be a solution of the differential equation

$$
F^{(k)}(t)+a_{k-1} F^{(k-1)}(t)+\cdots+a_{1} F^{\prime}(t)++B(r) F(t)=0
$$

where $t \in[0,1], a_{i}, i=1, \ldots, k-1$, are constants independent of $t$, and for some $K>0, B(r) \leq K r^{n+k}$, uniformly in $t$, for $r \geq r_{2}$ where $r_{2}$ was defined in Lemma 6. Let $g(r, t)$ be as defined in Lemma 6 , and that there exists a constant $c>1$ such that, for $m=0,1, \ldots, k-1$,

$$
\left|F^{(m)}(0)\right| \leq c g_{t}^{(m)}(r, 0)
$$


then there exists an $r_{3}$, where $r_{3}>r_{2}$ such that for all $r>r_{3}$

$$
\left|F^{(m)}(t)\right| \leq c g_{t}^{(m)}(r, t)
$$

for $t \in[0,1], m=0,1, \ldots, k-1$. The subscript $t$ of $g$ indicates that the differentiations are taken with respect to $t$ while $r$ is being kept fixed.

Proof. Let $c>1$ be a fixed constant such that (5.9) is satisfied, it is easy to check that $h(t):=c g(r, t)$ satisfies, as a function of $t$ with $r$ being kept fixed, the inequality

$$
\begin{aligned}
h^{(k)}-B_{k-1} h^{(k-1)}-\cdots-B_{1} h^{\prime}-B_{0}(r) h & \geq(c-1) \eta(r)^{k} \\
& =(c-1) N^{k} r^{n+k} \\
& \geq K r^{n+k}>0,
\end{aligned}
$$

where

$$
B_{j}:=\left|a_{j}\right|
$$

for $j=1, \ldots, k-1$; and

$$
B_{0}(r):=\left(\eta(r)^{k}-\left|a_{k-1}\right| \eta(r)^{k-1}-\cdots-\left|a_{1}\right| \eta(r)\right) \geq K r^{n+k}
$$

for all $r \geq r_{3}>r_{2}$ provided $N>0$ is chosen large enough. Now the conclusion follows at once from applying Lemma 3 to (5.8) and (5.11), plus the conditions (5.12) and (5.13).

Lemma 8. Let $p, \varepsilon>0$ and $\Delta>0$ be as defined in Theorem 4 , then there exist a constant $L=L(\varepsilon, \Delta)>1$ and a continuous path $\Lambda$ extending to infinity, consisting of radial intervals $\left(\Lambda_{j}\right)$ and circular arcs $\left(\Omega_{j}\right)$, which are contained in the set $\bigcup_{j} Q_{l, \varepsilon, H}\left(r_{1, j}, r_{2, j}\right)$ for certain $l$, as described in Theorem 4 and $\S 4$. Moreover, $\Lambda$ can be chosen so that it always keeps a distance at least $1 / L>0$ from the boundary of $\bigcup_{j} Q_{l, \varepsilon, H}\left(r_{1, j}, r_{2, j}\right)$. Suppose that there exist constants $K>0, n>0$ such that

$$
\left|A\left(r e^{i \theta}\right)\right| \leq K r^{n}, \quad r e^{i \theta} \in Q_{l, \varepsilon, H}\left(r_{1, j}, r_{2, j}\right),
$$

holds uniformly as $j \geq j_{0}(\varepsilon)$.

Then for any non-trivial solution $f(z)$ of (1.5) there exists a constant $A_{2}$ (depending only on $f$ and $\Delta$ ) such that

$$
\left|f\left(r e^{i \theta}\right)\right| \leq A_{2}^{j} j^{k j} \exp (\eta(r)(1+j))
$$

for $r e^{i \theta} \in Q_{l, \varepsilon, H}\left(r_{1, j}, r_{2, j}\right)$ and for all $j \geq j_{0}(\varepsilon)$ sufficiently large. 
Recalling the definition of the sectorial sets $\bigcup_{j} Q_{l, \varepsilon, H}\left(r_{1, j}, r_{2, j}\right)$ defined in $\S 4$, we next prove

Lemma 9. Let $p, \varepsilon>0$ and $\Delta>0$ be as defined in Theorem 4, and suppose that for some $l \in\{1, \ldots, p\},\left|z^{m} A\left(r e^{i \theta}\right)\right| \rightarrow 0$ uniformly in $\bigcup_{j} Q_{l, \varepsilon, H}\left(r_{1, j}, r_{2, j}\right)$, for each $m$, as $r \rightarrow \infty$. Then there exist a constant $L=L(\varepsilon, \Delta)>1$, and a fixed path

$$
\Lambda=\bigcup_{j \geq j_{0}}\left(\Lambda_{j} \cup \Omega_{j}\right) \subset \bigcup_{j} Q_{l, \varepsilon, H}\left(r_{1, j}, r_{2, j}\right)
$$

for some $l$, composed of radial intervals $\Lambda_{j}$ and circular arcs $\Omega_{j}$, extending to infinity, such that $\Lambda$ keeps away from the boundary of $\bigcup_{j} Q_{l, \varepsilon, H}\left(r_{1, j}, r_{2, j}\right)$ by a distance at least $1 / L>0$. For every solution $f$ of (1.5), there exists a constant $\gamma=\gamma(f)>0$ such that

$$
\left|f\left(r e^{i \theta}\right)\right| \leq \gamma^{4 s+2} r^{(2 s+2)(k-1)}
$$

for $r e^{i \theta} \in \Lambda_{j_{1}+s} \cup \Omega_{j_{1}+s}$, provided $j_{1}$ is sufficiently large. More precisely, we have, for all $s \geq 1$,

$$
\left|f\left(r e^{i \theta}\right)\right| \leq \gamma^{4 s} r^{(2 s+1)(k-1)} \quad \text { on } \Lambda_{j_{1}+s}
$$

and

$$
\left|f\left(r e^{i \theta}\right)\right| \leq \gamma^{4 s+2} r^{(2 s+2)(k-1)} \quad \text { on } \quad \Omega_{j_{1}+s} .
$$

\section{Proof of Lemma 8}

The idea of the proof is to construct a suitable path $\Lambda$ consisting of $\Lambda_{j}$ and $\Omega_{j}$ as in the statement of Lemma 8 and to apply Lemma 3 on $\Lambda_{j}, \Omega_{j}$ repeatedly for $j$ sufficiently large to construct comparison functions to estimate the growth of $f$ on $\Lambda_{j}$ and $\Omega_{j}$. The "comparison function" $g(r, t)$ is defined in Lemma 6 , and we work with it on approximating $f$ with respect to the independent variables $r$ and $t$ on $\Lambda_{j}$ and $\Omega_{j}$ respectively. In fact, as we enter from $Q_{l, \varepsilon, H}\left(r_{1, j}, r_{2, j}\right)$ to $Q_{l, \varepsilon, H}\left(r_{1, j+1}, r_{2, j+1}\right)$, we need to define, for each $j$, two functions $F$ and $g$ (the functions depend on some parameters which in turn depend on $j$ ) in order to make the argument work. Bearing in mind this convention, we shall simply write, with the same notations, $F$ and $g$ for every $j$ when it is clear from the context what the meaning of the comparison functions are. Since $\Delta$ is arbitrary, and so the angular measure of the exceptional set can be made arbitrary small, it is evident that we may construct the path $\Lambda$ to avoid $H$ and it may be chosen to have at most one turn in $Q_{l, \varepsilon, H}\left(r_{1, j}, r_{2, j}\right)$ on each $\Omega_{j}$ as stated in the Lemma. In fact, there are exactly two turnings in each $\Gamma_{j}$. But the second turning lies in the intersection of $\Gamma_{j}$ and $\Gamma_{j+1}$, and so it may be counted to lie in $\Gamma_{j+1}$, and the same argument applies to $\Gamma_{j+1}$ and $\Gamma_{j+2}$, etc. Thus we may assume, technically, that there is olny one 
turning in each $\Gamma_{j}$ in the actual computation. It is clear that there exists a positive constant $L=L(\varepsilon, \Delta)>0$ that the $\Lambda$ can be chosen so that it keeps away from the boundary of $\bigcup_{j} Q_{l, \varepsilon, H}\left(r_{1, j}, r_{2, j}\right)$ by a fixed positive constant $1 / L$, where $L>1$, for all $j \geq j_{0}(\varepsilon)$.

We proceed to the proof. Let $r e^{i \alpha_{j}}$ stand for a generic point on $\Lambda_{j}$ and we choose $j>j_{0}(\varepsilon)$ so large so that $r_{j_{0}}>r_{3}$ (recall that $r_{3}>r_{2}>r_{1}>r_{0}$ where $r_{0}$, $r_{1}, r_{2}$, and $r_{3}$ are defined in Lemmas $4,5,6,7$ respectively). Let $\alpha_{j}, \alpha_{j+1}, \ldots$ be the subsequent angles at which the path $\Lambda$ "turns". Assuming that there is a change of an angle from $\alpha_{j}$ to $\alpha_{j+1}$ for each $j$ as we are entering from $Q_{l, \varepsilon, H}\left(r_{1, j}, r_{2, j}\right)$ to $Q_{l, \varepsilon, H}\left(r_{1, j+1}, r_{2, j+1}\right)$, it is clear from the hypotheses that we may assume, from (4.5), that $0<\Delta<\left|\alpha_{j+1}-\alpha_{j}\right|<\varepsilon$ for each $j$ so that $\Lambda$ can always avoid the exceptional set $H$. In order to show our estimation remains valid for any configuration of $\Lambda$, we shall assume $\alpha_{j+1}<\alpha_{j}$ and $\alpha_{j+2}>\alpha_{j+1}$ as the initial values in the sequence of $\left\{\alpha_{s}\right\}$. We also write $|z|=r_{j}$ whenever $z \in \Omega_{j}$.

(a) Estimate on $\Omega_{j}$.

We assume $j \geq j_{0}$ is chosen sufficiently large so that the estimate in Lemma 4

$$
\left|f^{(m)}\left(r e^{i \alpha_{j}}\right)\right| \leq M \exp (\eta(r))
$$

holds for $m=1, \ldots, k-1$, where $\eta(r)$ is defined in Lemma 4 . We now define $\beta_{s+1}:=\beta_{s}+1$ for $s \geq j$ where $\beta_{j}=j$. Set

$$
g(r, t):=\exp \left(\eta(r)\left(t+\beta_{j}\right)\right)
$$

and

$$
F(t):=f\left(r e^{i \Theta}\right)
$$

where

$$
\Theta(t):=\alpha_{j}-t\left(\alpha_{j}-\alpha_{j+1}\right) .
$$

We deduce from (5.3) of Lemma 5 and (6.1) that, for $0 \leq t \leq 1$,

$$
\begin{aligned}
\left|F^{(m)}(0)\right| & \leq(2 \pi)^{k} D r_{j}^{m} \sum_{q=1}^{m}\left|f^{(q)}\left(r_{j} e^{i \Theta(0)}\right)\right| \\
& \leq(2 \pi)^{k} D M m r_{j}^{m} \exp \left(\eta\left(r_{j}\right)\left(0+\beta_{j}\right)\right) \\
& \leq(2 \pi)^{k} D k M \eta\left(r_{j}\right)^{m} \exp \left(\eta\left(r_{j}\right)(0+j)\right) \\
& =c_{j} g_{t}^{(m)}\left(r_{j}, 0\right),
\end{aligned}
$$

for $m=1, \ldots, k-1$ and where we have set

$$
c_{j}:=(2 \pi)^{k} k D M
$$


in (6.5). We now rewrite the equation $f^{(k)}+A f=0$ into a differential equation in $t$ of the form (5.8) with the aid of (5.5) where its coefficients $a_{i}, i=1, \ldots, k-1$, depend on $\alpha_{j+1}-\alpha_{j}$, but are otherwise fixed. The coefficient $B(r)$ in (5.8) becomes $\left(i v r e^{i \Theta}\right)^{k} A\left(r e^{i \Theta}\right)$. Clearly $c_{j}>1$ is the desired constant for $c$ in Lemma 7, and from (6.5) we deduce that (5.9) is satisfied. We obtain from (5.10)

$$
\left|F^{(m)}(t)\right| \leq c_{j} g_{t}^{(m)}(r, t)
$$

where $t \in[0,1]$ and $m=0,1, \ldots, k-1$.

(b). Estimate on $\Lambda_{j+1}$.

It follows from (a) above with $t=1$ and (5.4) that

$$
\begin{aligned}
\left|f^{(m)}\left(r_{j} e^{i \alpha_{j+1}}\right)\right| & \leq \frac{(2 \pi)^{m} D^{\prime}}{\left(\Delta r_{j}\right)^{m}} \sum_{q=1}^{m}\left|F^{(q)}(1)\right| \leq \frac{(2 \pi)^{m} D^{\prime}}{\left(\Delta r_{j}\right)^{m}} \sum_{q=1}^{m} c_{j} g_{t}^{(q)}\left(r_{j}, 1\right) \\
& =\frac{(2 \pi)^{m} D^{\prime}}{\left(\Delta r_{j}\right)^{m}} c_{j}\left(\eta\left(r_{j}\right)+\eta\left(r_{j}\right)^{2}+\cdots+\eta\left(r_{j}\right)^{m}\right) \exp \left(\eta\left(r_{j}\right)\left(1+\beta_{j}\right)\right) \\
& =\frac{(2 \pi)^{k} D^{\prime} c_{j}}{\Delta^{k}}\left(\frac{\eta\left(r_{j}\right)}{r_{j}}\right)^{m}\left(1+\cdots+\frac{1}{\eta\left(r_{j}\right)^{m-1}}\right) \exp \left(\eta\left(r_{j}\right)\left(1+\beta_{j}\right)\right) \\
& \leq \frac{2(2 \pi)^{k} D^{\prime} c_{j}}{\Delta^{k}}\left(\frac{\eta\left(r_{j}\right)}{r_{j}}\right)^{m} \exp \left(\eta\left(r_{j}\right)\left(0+\beta_{j+1}\right)\right) .
\end{aligned}
$$

Denoting

$$
d_{j}:=\frac{2(2 \pi)^{k} D^{\prime} c_{j}}{\Delta^{k}}
$$

and redefining $g(r, t)$ by

$$
g(r, t):=\exp \left(\eta(r)\left(t+\beta_{j+1}\right)\right)
$$

we have from $(6.8)$

$$
\left|f^{(m)}\left(r_{j} e^{i \alpha_{j+1}}\right)\right| \leq d_{j}\left(\frac{\eta\left(r_{j}\right)}{r_{j}}\right)^{m} g\left(r_{j}, 0\right)
$$

with the new parameter $\beta_{j+1}=\beta_{j}+1$. We prefer to use the same notation $g$ for this new function as it is clear from the context which definition we are working with. Thus (5.6) of Lemma 6 and (6.9) and (6.11) imply that, with this new $g$,

$$
\left|f^{(m)}\left(r_{j} e^{i \alpha_{j+1}}\right)\right| \leq d_{j}\left(\frac{\eta\left(r_{j}\right)}{r_{j}}\right)^{m} g\left(r_{j}, 0\right) \leq d_{j} g_{r}^{(m)}\left(r_{j}, 0\right)
$$

for $m=0,1, \ldots, k-1$. 
It is clear that $f\left(r e^{i \alpha_{j+1}}\right)$ solves the equation

$$
f_{r}^{(k)}\left(r e^{i \alpha_{j+1}}\right)-A_{0}(r) f\left(r e^{i \alpha_{j+1}}\right)=0
$$

where $A_{0}(r):=-e^{i k \alpha_{j+1}} A\left(r e^{i \alpha_{j+1}}\right)$ and the subscript $r$ indicates the $k$-th partial derivative is taken with respect to $r$. It follows from the proof of Lemma 6 that

$$
\begin{aligned}
\frac{g_{r}^{(k)}(r, 0)}{g(r, 0)} & =N^{k} \beta_{j+1}^{k}\left(1+\frac{n}{k}\right)^{k} r^{n}+O\left(r^{n-1}\right) \\
& \geq K r^{n} \geq\left|A\left(r e^{i \alpha_{j+1}}\right)\right|=A_{0}(r)
\end{aligned}
$$

on $\Lambda_{j+1}$ by the choice of $N$. Finally (6.12), (6.13), (6.14) and Lemma 3 give

$$
\left|f^{(m)}\left(r e^{i \alpha_{j+1}}\right)\right| \leq d_{j} g_{r}^{(m)}(r, 0)
$$

for $m=0,1, \ldots, k-1$ and $r e^{i \alpha_{j+1}} \in \Lambda_{j+1}$.

(c) Estimate on $\Omega_{j+1}$.

We define $F(t)=f\left(r e^{i \Theta}\right)$ as in (6.3), but with

$$
\Theta(t):=\alpha_{j+1}-t\left(\alpha_{j+1}-\alpha_{j+2}\right)
$$

where $\left|\alpha_{j+2}-\alpha_{j+1}\right| \geq \Delta>0$.

We deduce from (5.3) with $t=0,(5.6)$ and (6.15) that

$$
\begin{aligned}
\left|F^{(m)}(0)\right| & \leq(2 \pi)^{k} r_{j+1}^{m} D \sum_{q=1}^{m}\left|f^{(q)}\left(r_{j+1} e^{i \alpha_{j+1}}\right)\right| \leq(2 \pi)^{k} r_{j+1}^{m} D d_{j} \sum_{q=1}^{m} g_{r}^{(q)}\left(r_{j+1}, 0\right) \\
& \leq(2 \pi)^{k} D d_{j} m r_{j+1}^{m} g_{r}^{(m)}\left(r_{j+1}, 0\right) \\
& \leq(2 \pi)^{k} D d_{j} k r_{j+1}^{m}\left(A_{1}\left(2 \beta_{j+1}\right)^{m}\left(\frac{\eta\left(r_{j+1}\right)}{r_{j+1}}\right)^{m} g\left(r_{j+1}, 0\right)\right) \\
& \leq(2 \pi)^{k} k D A_{1}\left(2 \beta_{j+1}\right)^{k} d_{j} \eta\left(r_{j+1}\right)^{m} g\left(r_{j+1}, 0\right) \\
& =c_{j+1} \eta\left(r_{j+1}\right)^{m} \exp \left(\eta\left(r_{j+1}\right)\left(0+\beta_{j+1}\right)\right) \\
& =c_{j+1} g_{t}^{(m)}\left(r_{j+1}, 0\right)
\end{aligned}
$$

for $m=0,1, \ldots, k-1$, and where we have set

$$
c_{j+1}:=k D A_{1}(2 \pi)^{k}\left(2 \beta_{j+1}\right)^{k} d_{j}
$$

in (6.16). As in (a), we rewrite $f^{(k)}+A f=0$ into an equation of the form (5.8) where its coefficients $a_{i}, i=1, \ldots, k-1$ depend on $\left|\alpha_{j+2}-\alpha_{j+1}\right|$, but are otherwise fixed. We now argue as in (a) verbatim using Lemma 7 and with the new $g$ in (6.10) except to replace $\alpha_{j}, \alpha_{j+1}$ by $\alpha_{j+1}$ and $\alpha_{j+2}$, and $c_{j}$ by $c_{j+1}$ in (a). It follows from (6.6), 
(6.18) that $c_{j+1}>c_{j}$ and this together with (6.16), (5.9) and (5.10) of Lemma 7 give

$$
\left|F^{(m)}(t)\right| \leq c_{j+1} g_{t}^{(m)}\left(r_{j+1}, t\right)
$$

for $m=0,1, \ldots, k-1$ and $t \in[0,1]$.

(d) Estimate on $\Lambda_{j+2}$.

It follows from (6.19) we have (with the $F(t)$ associated with (6.16))

$$
\left|F^{(m)}(1)\right| \leq c_{j+1} g_{t}^{(m)}\left(r_{j+1}, 1\right)
$$

for $m=0,1, \ldots, k-1$. We deduce from $(6.19),(5.4)$ at $t=1$, and (6.10) that

$$
\begin{aligned}
\left|f^{(m)}\left(r_{j+1} e^{i \alpha_{j+2}}\right)\right| \leq \frac{(2 \pi)^{m} D^{\prime}}{\left(\Delta r_{j+1}\right)^{m}} \sum_{q=1}^{m}\left|F^{(q)}(1)\right| \\
\leq \frac{(2 \pi)^{m} D^{\prime}}{\left(\Delta r_{j+1}\right)^{m}} c_{j+1} \sum_{q=1}^{m} g_{t}^{(q)}\left(r_{j+1}, 1\right) \\
\leq \frac{(2 \pi)^{m} D^{\prime}}{\left(\Delta r_{j+1}\right)^{m}} c_{j+1}\left(\eta\left(r_{j+1}\right)+\cdots+\eta\left(r_{j+1}\right)^{m}\right) \exp \left(\eta\left(r_{j+1}\right)\left(1+\beta_{j+1}\right)\right) \\
\leq \frac{(2 \pi)^{m} D^{\prime} c_{j+1}}{\Delta^{m}}\left(\frac{\eta\left(r_{j+1}\right)}{r_{j+1}}\right)^{m}\left(1+\cdots+\frac{1}{\eta\left(r_{j+1}\right)^{m-1}}\right) \\
\quad \times \frac{2(2 \pi)^{k} D^{\prime} c_{j+1}}{\Delta^{k}}\left(\frac{\eta\left(r_{j+1}\right)}{r_{j+1}}\right)^{m} \exp \left(\eta\left(r_{j+1}\right)\left(0+1+\beta_{j+1}\right)\right) \\
=d_{j+1}\left(\frac{\eta\left(r_{j+1}\right)}{r_{j+1}}\right)^{m} g\left(r_{j+1}, 0\right)
\end{aligned}
$$

for $m=0,1, \ldots, k-1$, where

$$
d_{j+1}:=\frac{2(2 \pi)^{k} D^{\prime} c_{j+1}}{\Delta^{k}}
$$

in (6.20) and the new function $g$ in the last equality of (6.20) is now defined by

$$
g(r, t):=\exp \left(\eta(r)\left(t+\beta_{j+2}\right)\right)
$$

where $\beta_{j+2}:=1+\beta_{j+1}$. We deduce as in part (b) with (5.6) and (6.20) that

$$
\left|f^{(m)}\left(r_{j+1} e^{i \alpha_{j+2}}\right)\right| \leq d_{j+1}\left(\frac{\eta\left(r_{j+1}\right)}{r_{j+1}}\right)^{m} g\left(r_{j+1}, 0\right) \leq d_{j+1} g_{r}^{(m)}\left(r_{j+1}, 0\right)
$$

for $m=0,1, \ldots, k-1$. 
As in (b), $f\left(r e^{i \alpha_{j+2}}\right)$ solves the equation

$$
f_{r}^{(k)}\left(r e^{i \alpha_{j+2}}\right)-A_{0}(r) f\left(r e^{i \alpha_{j+2}}\right)=0
$$

where $A_{0}(r):=-e^{-i k \alpha_{j+2}} A\left(r e^{i \alpha_{j+2}}\right)$ where we have used the same notation $A_{0}(r)$ as in case (b) while the meaning of it is clear from the context.

We apply the same argument as in (b) with $\beta_{j+1}$ replaced by $\beta_{j+2}$ and for $z \in \Lambda_{j+2}, j \geq j_{0}(\varepsilon)$, We have

$$
\begin{aligned}
\frac{g_{r}^{(k)}(r, 0)}{g(r, 0)} & =\left(N \beta_{j+2}\left(1+\frac{n}{k}\right)\right)^{k} r^{n}+O\left(r^{n-1}\right) \geq K r^{n} \geq\left|A\left(r e^{i \alpha_{j+2}}\right)\right| \\
& =A_{0}(r) .
\end{aligned}
$$

It follows from $(6.23),(6.24),(6.25)$ and Lemma 3 that

$$
\left|f^{(m)}\left(r e^{i \alpha_{j+2}}\right)\right| \leq d_{j+1} g_{r}^{(m)}(r, 0)
$$

for $m=0,1, \ldots, k-1$ and $r e^{i \alpha_{j+2}} \in \Lambda_{j+2}$.

As the argument above does not depend on the particular sequence $\left\{\alpha_{j}\right\}$, we may continue the process $(\mathrm{a}-\mathrm{d})$ above repeatedly, and by induction the estimate remains valid for the most general $\Lambda$ as described in the Lemma.

(e) Computation of constants.

From (6.6) of (a) above, we have

$$
c_{j}:=(2 \pi)^{k} k D M
$$

and from (6.9) we write

$$
d_{j}:=\frac{2(2 \pi)^{k} D^{\prime}}{\Delta^{k}} c_{j}:=\tau c_{j}
$$

We set $\kappa:=k D A_{1}(2 \pi)^{k} 2^{k}$ and this with the above notations and (6.18) give

$$
c_{j+1}:=k D A_{1}(2 \pi)^{k}\left(2 \beta_{j+1}\right)^{k} d_{j}=\kappa \beta_{j+1}^{k} d_{j}=\kappa \tau \beta_{j+1}^{k} c_{j} .
$$

We have defined in $(6.21) d_{j+1}:=\frac{2(2 \pi)^{k} D^{\prime}}{\Delta^{k}} c_{j+1}:=\tau c_{j+1}$ and this yields, as in the pervious part,

$$
c_{j+2}:=\kappa \beta_{j+2}^{k} d_{j+1}=\kappa \tau \beta_{j+2}^{k} c_{j+1}=(\kappa \tau)^{2}\left(\beta_{j+1} \beta_{j+2}\right)^{k} c_{j} .
$$

It follows from induction and the definition of $\beta_{j}=j$

$$
\begin{aligned}
c_{j+s}:=(\kappa \tau)^{s}\left(\beta_{j+1} \beta_{j+2} \ldots \beta_{j+s}\right)^{k} c_{j} & \leq(\kappa \tau)^{s} \beta_{j+s}^{k s} c_{j} \\
& =(\kappa \tau)^{s}(j+s)^{k s} c_{j}
\end{aligned}
$$


for all $s \geq 1$, since $\beta_{j+s}:=s+\beta_{j}=j+s$.

Similarly, we have $d_{j}=\tau c_{j}$ and $d_{j+1}:=\tau c_{j+1}=\tau \kappa \beta_{j+1}^{k} d_{j}$. We work out one more term:

$$
\begin{aligned}
d_{j+2}:=\tau c_{j+2} & =\tau\left(\kappa \tau \beta_{j+2}^{k} c_{j+1}\right) \\
& =\tau \kappa \tau \beta_{j+2}^{k}\left(\tau \kappa \beta_{j+1}^{k} c_{j}\right) \\
& =\kappa \tau \beta_{j+2}^{k} \tau \kappa \beta_{j+1}^{k} d_{j} \\
& =(\kappa \tau)^{2}\left(\beta_{j+1} \beta_{j+2}\right)^{k} d_{j} .
\end{aligned}
$$

By induction, we easily obtain from (6.27-9)

$$
\begin{aligned}
d_{j+s}: & =(\kappa \tau)^{s}\left(\beta_{j+1} \beta_{j+2} \ldots \beta_{j+s}\right)^{k} d_{j} \\
& \leq(\kappa \tau)^{s}\left(\beta_{j+s}\right)^{k s} d_{j} \\
& =(\kappa \tau)^{s}(j+s)^{k s} d_{j},
\end{aligned}
$$

for all $s \geq 1$ and $j \geq j_{0}(\varepsilon)$.

Combining and renumbering $j+s$ in (6.29) and (6.30) we obtain the required estimate, i.e., there exists a constant $A_{2}$ such that

$$
\left|f\left(r e^{i \theta}\right)\right| \leq A_{2}^{j} j^{k j} \exp (\eta(r)(t+j)) \leq A_{2}^{j} j^{k j} \exp (\eta(r)(1+j))
$$

for $z \in \Lambda$ and all $j$ sufficiently large.

REMARK. Since the estimate of $f\left(r_{j} e^{i \alpha_{j}}\right)$ at $r_{j} e^{i \alpha_{j}}$ on $\Omega_{j}$ for any $j$ can proceed in clockwise or counter-clockwise direction, the estimates in the Lemma can be extended on the whole arc containing $\Omega_{j}$, which is contained in $Q_{l, \varepsilon, H}\left(r_{1, j}, r_{2, j}\right)$. We shall use this fact in the proof of Theorem 4 later in $\S 8$.

\section{Proof of Lemma 9}

By using the same notation as in Lemma 8, we may conclude as in the beginning of Section 6 the existence of $\Lambda$ in $\bigcup_{j} Q_{l, \varepsilon, H}\left(r_{1, j}, r_{2, j}\right)$ such that $\Lambda$ keeps away from the boundary of $\bigcup_{j} Q_{l, \varepsilon, H}\left(r_{1, j}, r_{2, j}\right)$ by a fixed constant $1 / L$ where $L=L(\varepsilon, \Delta)>1$ and for $j \geq j_{1}:=j_{1}(\varepsilon)$ for a suitably chosen $j_{1}$, and $\Lambda$ can be regarded to have at most one turn in each $Q_{l, \varepsilon, H}\left(r_{1, j}, r_{2, j}\right)$. As our argument below does not depend on the particular sequence of $\left\{\alpha_{s}\right\}$, we may assume without loss of generality that the sequence is monotone; we write $|z|=r_{j}$ for $z \in \Omega_{j}$ for all $j$. We shall use induction to prove this lemma.

Let $z_{j}^{*}=r_{j} e^{i \alpha_{j}}$ be the upper end point of $\Lambda_{j}, z_{s+1}=r_{s} e^{i \alpha_{s+1}}$ and $z_{s+1}^{*}=$ $r_{s+1} e^{i \alpha_{s+1}}$ be the lower and upper end points of $\Lambda_{s+1}$ respectively, for $s \geq j \geq j_{1}$. 
According to the hypotheses we may assume that $j \geq j_{1} \geq j_{0}$ (where $j_{0}$ is as in Lemma 8) is chosen so large that

$$
\frac{2^{k} \pi}{(k-1) !} r^{k+1}\left|A\left(r e^{i \theta}\right)\right|<1 / 2
$$

for all $z \in \Lambda$. Then we may fix a constant $B>1$ such that

$$
\left|f\left(r e^{i \alpha_{j_{1}}}\right)\right| \leq B r^{k-1}
$$

holds for $z$ on $\Lambda_{j_{1}}$.

(a) Estimate on $\Lambda_{j_{1}}$.

Let $z_{j_{1}}=r_{j_{1}} e^{i \alpha_{j_{1}}}$ be the lower end point of $\Lambda_{j_{1}}$. Write (5.1) as

$$
\begin{aligned}
f(z)=c_{0}+c_{1}\left(z-z_{j_{1}}\right)+\cdots+c_{k-1}\left(z-z_{j_{1}}\right)^{k-1} \\
-\frac{1}{(k-1) !} \int_{z_{j_{1}}}^{z}(z-\tau)^{k-1} A(\tau) f(\tau) d \tau
\end{aligned}
$$

where

$$
c_{0}=f\left(z_{j_{1}}\right), \quad c_{1}=f^{\prime}\left(z_{j_{1}}\right), \ldots, \quad c_{k-1}=f^{(k-1)}\left(z_{j_{1}}\right) /(k-1) !
$$

and the integration is taken on $\Lambda_{j_{1}}$ from $z_{j_{1}}$ to $z=r e^{i \alpha_{j_{1}}} \in \Lambda_{j_{1}}$ and denote

$$
c:=\max _{0 \leq q \leq k-1}\left(\left|c_{q}\right|\right)
$$

Defining

$$
\gamma_{1}:=B L^{k}, \quad \gamma_{2}:=k 2^{k-1} e^{1 / 2}, \quad \gamma:=\gamma_{1} \gamma_{2},
$$

we see that it follows from (7.3) that

$$
|f(z)|=\left|f\left(r e^{i \alpha_{j_{1}}}\right)\right| \leq B r^{k-1}<\gamma_{1} r^{k-1}<\gamma r^{k-1} .
$$

It follows from (7.7) and Cauchy's estimate, for $z$ on $\Lambda_{j_{1}}$ that

$$
\left|f^{(m)}(z)\right| \leq \max _{2 \leq m \leq k}\left(L^{m}\right) B r^{k-1} \leq \gamma_{1} r^{k-1}<\gamma r^{k-1} .
$$

In particular, when $z=z_{j_{1}}^{*}$, we have

$$
\left|f^{(m)}\left(z_{j_{1}}^{*}\right)\right| \leq \gamma r_{j_{1}}^{k-1}
$$

for $m=1, \ldots, k-1$. 
(b) Estimate on $\Omega_{j_{1}}$.

Write now (5.1) as

$$
\begin{aligned}
f(z)=c_{0, j_{1}}+ & c_{1, j_{1}}\left(z-z_{j_{1}}^{*}\right)+\cdots+c_{k-1, j_{1}}\left(z-z_{j_{1}}^{*}\right)^{k-1} \\
& -\frac{1}{(k-1) !} \int_{z_{j_{1}}^{*}}^{z}(z-\tau)^{k-1} A(\tau) f(\tau) d \tau
\end{aligned}
$$

where

$$
c_{0, j_{1}}=f\left(z_{j_{1}}^{*}\right), \quad c_{1, j_{1}}=f^{\prime}\left(z_{j_{1}}^{*}\right), \quad \ldots, \quad c_{k-1, j_{1}}=f^{(k-1)}\left(z_{j_{1}}^{*}\right) /(k-1) !,
$$

and the integration is taken on $\Omega_{j_{1}}$ (with respect to $t$; see below). Notice that one obtains an estimate on $\left|c_{q, j_{1}}\right|$ from (7.11).

Writing $\Theta(t):=\alpha_{j_{1}}-t\left(\alpha_{j_{1}}-\alpha_{j_{1}+1}\right), 0 \leq t \leq 1$ and applying the Gronwall lemma $[14$, p. 86$]$ to $(7.10)$ with respect to $t$, then $(7.6),(7.1),(7.9)$ and (7.11) give

$$
\begin{aligned}
\left|f\left(r_{j_{1}} e^{i \Theta}\right)\right| \leq & \max _{0 \leq q \leq k-1}\left(\left|c_{q, j_{1}}\right|\right)\left(2 r_{j_{1}}\right)^{k-1} \\
& \quad+\frac{1}{(k-1) !} \int_{0}^{t}\left(2 r_{j_{1}}\right)^{k-1}\left|A\left(r_{j_{1}} e^{i \Theta}\right) f\left(r_{j_{1}} e^{i \Theta}\right)\right| r_{j_{1}}\left|\alpha_{j_{1}}-\alpha_{j_{1}+1}\right| d t \\
\leq & k 2^{k-1}\left(\gamma r_{j_{1}}^{k-1}\right) r_{j_{1}}^{k-1}+\left\{\frac{2^{k} \pi}{(k-1) !} \int_{0}^{t} r_{j_{1}}^{k}\left|A\left(r_{j_{1}} e^{i \Theta(t)}\right) f\left(r_{j_{1}} e^{i \Theta(t)}\right)\right| d t\right\} \\
\leq & k 2^{k-1} \gamma r_{j_{1}}^{2(k-1)} \exp \left\{\frac{2^{k} \pi}{(k-1) !} \int_{0}^{t} r_{j_{1}}^{k}\left|A\left(r_{j_{1}} e^{i \Theta(t)}\right)\right| d t\right\} \\
\leq & k 2^{k-1} e^{1 / 2} \gamma r_{j_{1}}^{2(k-1)} \leq \gamma_{2} \gamma r_{j_{1}}^{2(k-1)} \leq \gamma^{2} r_{j_{1}}^{2(k-1)} .
\end{aligned}
$$

In particular, for $t=1$ we get

$$
\left|f\left(z_{j_{1}+1}\right)\right|=\left|f\left(r_{j_{1}} e^{i \alpha_{j_{1}+1}}\right)\right| \leq \gamma^{2} r_{j_{1}}^{2(k-1)} .
$$

By Cauchy's estimate and (7.12) we see on $\Omega_{j_{1}}$ that

$$
\left|f^{(m)}\left(r_{j_{1}} e^{i \Theta}\right)\right| \leq L^{k} \gamma^{2} r_{j_{1}}^{2(k-1)} \leq \gamma_{1} \gamma^{3} r_{j_{1}}^{2(k-1)}<\gamma^{3} r_{j_{1}}^{2(k-1)} .
$$

Hence

$$
\left|f^{(m)}(z)\right| \leq \gamma^{3} r_{j_{1}}^{2(k-1)}
$$

for $z \in \Omega_{j_{1}}$ and $m=1, \ldots, k-1$. In particular

$$
\left|f^{(m)}\left(z_{j_{1}+1}\right)\right|=\left|f^{(m)}\left(r_{j_{1}} e^{i \alpha_{j_{1}+1}}\right)\right| \leq \gamma^{3} r_{j_{1}}^{2(k-1)}
$$

for $m=1, \ldots, k-1$. 
(c) Inductive hypotheses on $\Omega_{j+s-1}$ and $\Lambda_{j+s}$.

We denote $z_{j+s}=r_{j_{1}+s-1} e^{i \alpha_{j_{1}+s}}$ and $z_{j+s}^{*}=r_{j_{1}+s} e^{i \alpha_{j_{1}+s}}$ be the lower and upper end points of $\Lambda_{j+s}$. Similarly we set $z_{j_{1}+s}^{*}=r_{j_{1}+s} e^{i \alpha_{j_{1}+s}}$ and $z_{j_{1}+s+1}=$ $r_{j_{1}+s} e^{i \alpha_{j_{1}+s+1}}$ be the end points of $\Omega_{j_{1}+s}$.

Assume now that the following estimates hold on $z \in \Omega_{j_{1}+s-1}$ :

$$
|f(z)| \leq \gamma^{4 s-2} r_{j_{1}+s-1}^{2 s(k-1)}
$$

and

$$
\left|f^{(m)}(z)\right| \leq \gamma^{4 s-1} r_{j_{1}+s-1}^{2 s(k-1)}
$$

for $m=1, \ldots, k-1$; while for $z=r e^{i \alpha_{j_{1}+s}} \in \Lambda_{j_{1}+s}$ we have

$$
|f(z)| \leq \gamma^{4 s} r^{(2 s+1)(k-1)} \leq \gamma^{4 s} r_{j_{1}+s}^{(2 s+1)(k-1)}
$$

and

$$
\left|f^{(m)}(z)\right| \leq \gamma^{4 s+1} r^{(2 s+1)(k-1)} \leq \gamma^{4 s+1} r_{j_{1}+s}^{(2 s+1)(k-1)},
$$

by Cauchy's estimate, for $m=1, \ldots, k-1$.

(d) Estimate on $\Omega_{j_{1}+s}$.

We have, by (5.1)

$$
\begin{array}{r}
f(z)=c_{0, j_{1}+s}+c_{1, j_{1}+s}\left(z-z_{j_{1}+s}^{*}\right)+\cdots+c_{k-1, j_{1}+s}\left(z-z_{j_{1}+s}^{*}\right)^{k-1} \\
-\frac{1}{(k-1) !} \int_{z_{j_{1}+s}}^{z}(z-\tau)^{k-1} A(\tau) f(\tau) d \tau
\end{array}
$$

where

$$
\begin{aligned}
& c_{0, j_{1}+s}=f\left(z_{j_{1}+s}^{*}\right), \quad c_{1, j_{1}+s}=f^{\prime}\left(z_{j_{1}+s}^{*}\right), \ldots, \\
& c_{k-1, j_{1}+s}=f^{(k-1)}\left(z_{j_{1}+s}^{*}\right) /(k-1) !
\end{aligned}
$$

and the integration is taken along $\Omega_{j_{1}+s}$. Writing $\Theta(t):=\alpha_{j_{1}+s}-t\left(\alpha_{j_{1}+s}-\alpha_{j_{1}+s+1}\right)$ and applying the Gronwall lemma to (7.21) with respect to $t$, it follows from (7.22), (7.20), (7.19), (7.6) and (7.1), for $z=r_{j_{1}+s} e^{i \Theta} \in \Omega_{j_{1}+s}$, that

$$
\begin{aligned}
& \left|f\left(r_{j_{1}+s} e^{i \Theta}\right)\right| \\
& \quad \leq k \max _{\mu}\left(\left|c_{\mu, j_{1}+s}\right|\right)\left(2 r_{j_{1}+s}\right)^{k-1} \\
& \quad+\frac{1}{(k-1) !} \int_{0}^{t}\left(2 r_{j_{1}+s}\right)^{k-1}\left|A\left(r_{j_{1}+s} e^{i \Theta}\right) f\left(r_{j_{1}+s} e^{i \Theta}\right)\right| r_{j_{1}+s}\left|\alpha_{j_{1}+s}-\alpha_{j_{1}+s+1}\right| d t \\
& \quad \leq k 2^{k-1}\left(\gamma^{4 s+1} r_{j_{1}+s}^{(2 s+1)(k-1)}\right) r_{j_{1}+s}^{k-1}+\frac{2^{k-1} 2 \pi}{(k-1) !} \int_{0}^{t} r_{j_{1}+s}^{k}\left|A\left(r_{j_{1}+s} e^{i \Theta}\right) f\left(r_{j_{1}+s} e^{i \Theta}\right)\right| d t
\end{aligned}
$$




$$
\begin{aligned}
& \leq k 2^{k-1} \gamma^{4 s+1} r_{j_{1}+s}^{(2 s+2)(k-1)} \exp \left\{\frac{2^{k} \pi}{(k-1) !} \int_{0}^{t} r_{j_{1}+s}^{k}\left|A\left(r_{j_{1}+s} e^{i \Theta}\right)\right|\right\} d t \\
& \leq k 2^{k-1} e^{1 / 2} \gamma^{4 s+1} r_{j_{1}+s}^{(2 s+2)(k-1)}<\gamma_{1} \gamma^{4 s+1} r_{j_{1}+s}^{(2 s+2)(k-1)} \leq \gamma^{4 s+2} r_{j_{1}+s}^{(2 s+2)(k-1)} .
\end{aligned}
$$

In particular, we have

$$
\left|f\left(r_{j_{1}+s}^{i \alpha_{j_{1}+s+1}}\right)\right| \leq \gamma^{4 s+2} r_{j_{1}+s}^{(2 s+2)(k-1)}
$$

It follows from $(7.23),(7.6)$ and Cauchy's estimate that

$$
\begin{aligned}
\left|f^{(m)}\left(r_{j_{1}+s} e^{i \theta}\right)\right| & \leq L^{k} \gamma^{4 s+2} r_{j_{1}+s}^{(2 s+2)(k-1)}<\gamma_{1} \gamma^{4 s+2} r_{j_{1}+s}^{(2 s+2)(k-1)} \\
& \leq \gamma^{4 s+3} r_{j_{1}+s}^{(2 s+2)(k-1)}
\end{aligned}
$$

for $m=1, \ldots, k-1$. In particular, we obtain

$$
\left|f^{(m)}\left(z_{j_{1}+s+1}\right)\right|=\left|f^{(m)}\left(r_{j_{1}+s} e^{i \alpha_{j_{1}+s+1}}\right)\right| \leq \gamma^{4 s+3} r_{j_{1}+s}^{(2 s+2)(k-1)}
$$

for $m=0,1, \ldots, k-1$.

(e) Estimate on $\Lambda_{j_{1}+s+1}$.

As in (d), we have

$$
\begin{gathered}
f(z)=c_{0, j_{1}+s+1}+c_{1, j_{1}+s+1}\left(z-z_{j_{1}+s+1}\right)+\cdots+c_{k-1, j_{1}+s+1}\left(z-z_{j_{1}+s+1}\right)^{k-1} \\
-\frac{1}{(k-1) !} \int_{z_{j_{1}+s+1}}^{z}(z-\tau)^{k-1} A(\tau) f(\tau) d \tau
\end{gathered}
$$

where

$$
\begin{gathered}
c_{0, j_{1}+s+1}=f\left(z_{j_{1}+s+1}\right), \quad c_{1, j_{1}+s+1}=f^{\prime}\left(z_{j_{1}+s+1}\right), \ldots \\
c_{k-1, j_{1}+s+1}=f^{(k-1)}\left(z_{j_{1}+s+1}\right) /(k-1) !
\end{gathered}
$$

and the integration is along $\Lambda_{j_{1}+s+1}$. It follows from $(7.27),(7.28),(7.26),(7.1)$ and (7.6) and the Gronwall lemma that

$$
\begin{aligned}
\left|f\left(r e^{i \alpha_{j_{1}+s+1}}\right)\right| \leq & k \max _{\mu}\left(\left|c_{\mu, j_{1}+s+1}\right|\right)(2 r)^{k-1} \\
& +\frac{2^{k-1}}{(k-1) !} \int_{r_{j_{1}+s+1}}^{r} r^{k-1}\left|A\left(t e^{i \alpha_{j_{1}+s+1}}\right) f\left(t e^{i \alpha_{j_{1}}+s+1}\right)\right| d t \\
\leq & k 2^{k-1}\left(\gamma^{4 s+3} r_{j_{1}+s}^{(2 s+2)(k-1)}\right) r^{k-1} \\
& \quad \times \exp \left\{\frac{2^{k-1}}{(k-1) !} \int_{r_{j_{1}+s+1}}^{r} r^{k-1}\left|A\left(t e^{i \alpha_{j_{1}+s+1}}\right)\right| d t\right\}
\end{aligned}
$$




$$
\begin{aligned}
& \leq k 2^{k-1} e^{1 / 2} \gamma^{4 s+3} r^{(2 s+3)(k-1)} \leq \gamma_{2} \gamma^{4 s+3} r^{(2 s+3)(k-1)} \\
& <\gamma^{4 s+4} r^{(2 s+3)(k-1)}=\gamma^{4(s+1)} r^{(2(s+1)+1)(k-1)} .
\end{aligned}
$$

In particular, we get

$$
\left|f\left(r_{j_{1}+s+1} e^{i \alpha_{j_{1}+s+1}}\right)\right| \leq \gamma^{4(s+1)} r_{j_{1}+s+1}^{(2(s+1)+1)(k-1)} .
$$

By the Cauchy estimate and (7.29) we obtain

$$
\begin{aligned}
\left|f^{(m)}\left(r e^{i \alpha_{j_{1}+s+1}}\right)\right| & \leq L^{k} \gamma^{4(s+1)} r^{(2 s+3)(k-1)}<\gamma_{1} \gamma^{4(s+1)} r^{(2 s+3)(k-1)} \\
& <\gamma^{4 s+5} r^{(2 s+3)(k-1)} \\
& \leq \gamma^{4(s+1)+1} r^{(2(s+1)+1)(k-1)}
\end{aligned}
$$

for $m=1, \ldots, k-1$. In particular,

$$
\left|f^{(m)}\left(r_{j_{1}+s+1}^{i \alpha_{j_{1}}+s+1}\right)\right| \leq \gamma^{4 l+5} r_{j_{1}+s+1}^{(2 s+3)(k-1)}=\gamma^{4(s+1)+1} r_{j_{1}+s+1}^{(2(s+1)+1)(k-1)}
$$

for $m=0,1, \ldots, k-1$.

Hence (7.17-7.20) hold for all $s \geq 1$ and $j \geq j_{1}(\varepsilon)$, and we have the lemma.

Remark. Although the estimate was only proved to be valid on $\Lambda$, and in particular on $\Omega_{j}$, the estimate on $\Omega_{j}$ is such that it is independent of the orientation, and so it is clear that the estimate there can be extended to the whole arc in $Q_{l, \varepsilon, H}\left(r_{1, j}, r_{2, j}\right)$ containing $\Omega_{j}$.

\section{Proof of Theorem 4}

We still require a few more facts. All of them can be found in [6]. Firstly, if $f=\pi e^{h}$ is a non-trivial solution of (1.5) with $\lambda(f)<\infty$, then $h(z)$ must be an entire function of finite order (see also [3], p. 11). Secondly, if $f_{1}, \ldots, f_{k}$ is a fundamental set of (1.5) each with $\lambda\left(f_{i}\right)<\infty$, then the product $E=f_{1} \cdots f_{k}$ is of finite order ([6] Theorem 1). If in addition that $\lambda\left(f_{i}\right)<\sigma(A), i=1, \ldots, k$, then $\sigma(E)=\sigma(A) \in \mathbb{N}([6]$ Theorem 3). Therefore, we must have $\sigma(E) \geq \sigma(A)$ as soon as each $\lambda\left(f_{i}\right)<\infty$. See also the remark after Theorem 3 .

Finally, we note that Bank and Langley proved (see [6], Theorem 2) that if $\rho(A)<1 / 2$, then the equation (1.5) cannot have two linearly independent solutions $f_{1}, f_{2}$ each with finite exponent of convergence. Hence we may assume that $\sigma(A) \geq$ $1 / 2$.

For easy understanding, we outline the main idea of our proof. Assuming the contrary, we suppose each $\lambda\left(f_{i}\right)$ is finite and thus $E:=f_{1} f_{2} \cdots f_{k}$ has finite exponent of convergence (and hence $E$ must be of finite order no less than $\sigma(A)$ mentioned 
in the first paragraph above). Hence it suffices to show $\sigma(E)<\sigma(A)$, and this will give a contradiction. We shall incorporate the ideas used in [6] and [8] in our proof. However, our argument is more complicated. In order to obtain the estimate on $T(r, E)$, we first estimate the growth of $E$ along certain paths $\Lambda$ (instead of rays in [6]) within each of the connected region $\bigcup_{j} Q_{l, \varepsilon, H}\left(r_{1, j}, r_{2, j}\right)$ for $l=1, \ldots, p$ (depending on whether $A(z)$ satisfies (i), (ii) or (iii) of the hypotheses) and then obtain estimates on circles passing through these connected regions (Lemmas 8 and $9)$.

We now proceed to the proof. Given $\varepsilon>0$, there exists $j_{2}=j_{2}(\varepsilon)$ such that $j_{2} \geq \max \left(j_{0}, j_{1}\right)$, where $j_{0}, j_{1}$ are defined in Lemmas 8 and 9 respectively, and there exist, for each $j \geq j_{2}, \theta_{1}<\theta_{2}<\cdots<\theta_{p}$ (we remind the reader that we have dropped the dependence of $\theta_{i}$ on $j$ for the sake of brevity) and two sequences $\left\{r_{1, j}\right\},\left\{r_{2, j}\right\}$ so that $A(z)$ satisfies either (i), (ii) or (iii) of the hypotheses. It follows from Lemmas 8 and 9 that, for each $l \in\{1, \ldots, p\}$, there exists a path $\Lambda$ passing through $\bigcup_{j} Q_{l, \varepsilon, H}\left(r_{1, j}, r_{2, j}\right)$ which is defined in $\S 4$. Any such $\Lambda$ can be chosen so that it keeps a distance $1 / L>0$ away from the boundary of $\bigcup_{j} Q_{l, \varepsilon, H}\left(r_{1, j}, r_{2, j}\right)$.

We proceed to estimate $T(r, E)$ outside a set $H$ of $\underline{\log \underline{\operatorname{dens}}} H_{P}=0$. Note that it may be necessary at some later stages to apply Lemma 1 outside of an exceptional set of finite linear measure (or indeed an $R$-set). Since such an exceptional set is of upper logarithmic density zero, we may therefore incorporate these exceptional set into $H$, and assume without loss of generality that any chosen $r$ below lies outside these exceptional sets which we shall assume throughout.

Since $j \geq j_{2}(\varepsilon)$ and the size of the exceptional set can be arbitrary small (since $\Delta>0$ can be chosen to be arbitrarily small), we may select the radius of $\Omega_{j}$ for each $l \in\{1, \ldots, p\}$ to be the same. Hence we choose a circle of integration for $T(r, E)$ such that its radius is common to those of the circular arcs $\Omega_{j}$ on which both Lemmas 8 and 9 hold simultaneously. Now consider $T(r, E)$ so that $r$ is as described above, and we may write it as

$$
\int_{0}^{2 \pi} \log \left|E\left(r e^{i \theta}\right)\right| d \theta=\sum_{l=1}^{p} \int_{Q_{l, \varepsilon}} \log ^{+}\left|E\left(r e^{i \theta}\right)\right| d \theta+\sum_{l=1}^{p} \int_{P_{l, \varepsilon}} \log ^{+}\left|E\left(r e^{i \theta}\right)\right| d \theta
$$

for a fixed $r \in\left(r_{1, j}, r_{2, j}\right) \backslash H_{P}$ and $j \geq j_{2}(\varepsilon)$, say.

Since the growth of $A(z)$ which is given by (i), (ii) and (iii) across the $Q_{l, \varepsilon, H}\left(r_{1, j}, r_{2, j}\right)(l=1, \ldots, p)$ is uniform, it is therefore sufficient in the estimation of (8.1) to consider the following terms

$$
\int_{Q_{l, \varepsilon}} \log ^{+}\left|E\left(r e^{i \theta}\right)\right| d \theta+\int_{P_{l+1, \varepsilon}} \log ^{+}\left|E\left(r e^{i \theta}\right)\right| d \theta:=I_{1}+I_{2}
$$

where $r e^{i \theta} \in Q_{l, \varepsilon, H}\left(r_{1, j}, r_{2, j}\right), P_{l+1, \varepsilon}\left(r_{1, j}, r_{2, j}\right), l=1, \ldots, p$, respectively, and where $j \geq j_{2}(\varepsilon)$ is sufficiently large. The remaining terms for $Q_{l, \varepsilon}, P_{l, \varepsilon}$ are similar. 
We split the proof into two parts, estimating first $I_{1}$ in Part A, and then $I_{2}$ in Part B. Here we work on the smaller regions $Q_{l, 2 \varepsilon}$ instead of $Q_{l, \varepsilon}$.

Part A. Subcase I: $A(z)$ satisfies (i) of Theorem 4. Suppose $f_{i}=\pi_{i} e^{h_{i}}, i=$ $1, \ldots k$, is a fundamental set of (4.12). Substituting $f_{i}=\pi_{i} e^{h_{i}}$ into equation (4.12) yields

$$
\left(h_{i}^{\prime}\right)^{k}+\sum_{\mu=0}^{k-1} B_{\mu}(z)\left(h_{i}^{\prime}\right)^{\mu}+A(z)=0
$$

where $B_{\mu}(z)$ is a polynomial in $\pi_{i}^{\prime} / \pi_{i}, h_{i}^{\prime \prime} / h_{i}^{\prime}$ and their derivatives with constant coefficients. But $\pi(z)$ and $h(z)$ are both of finite order (see the paragraph before Lemma 1), and so

$$
\left(\left|\frac{\pi_{i}^{\prime}}{\pi_{i}}\right|+\cdots+\left|\frac{\pi_{i}^{(k)}}{\pi_{i}}\right|\right)+\left(\left|\frac{h_{i}^{\prime \prime}}{h_{i}^{\prime}}\right|+\cdots+\left|\frac{h_{i}^{(k)}}{h_{i}^{\prime}}\right|\right) \leq|z|^{M_{1}}
$$

for some $M_{1}>0$ for all $r$ outside a set of finite linear measure (in fact, outside an $R$-set), see [14], Proposition 5.12. Let us rewrite the equation (8.3) into the following form:

$$
\left(\frac{h_{i}^{\prime}}{A^{1 / k}}\right)^{k}+\sum_{\mu=0}^{k-1}\left(\frac{B_{\mu}}{A^{\frac{k-\mu}{k}}}\right)\left(\frac{h_{i}^{\prime}}{A^{1 / k}}\right)^{\mu}+1=0 .
$$

According to the hypothesis about $H_{P}$, it is possible to choose $r_{1, j}, R_{2, j}$, such that $r_{1, j}<R_{1, j}<R_{2, j}<r_{2, j}$ and $R_{2, j}-R_{1, j} \not \rightarrow 0$. Hence the set defined by $Q_{l, 2 \varepsilon, H}\left(R_{1, j}, R_{2, j}\right)$ is a subset of $Q_{l, \varepsilon, H}\left(r_{1, j}, r_{2, j}\right)$, where $r_{1, j}<R_{1, j}<R_{2, j}<r_{2, j}$, such that there are no exceptional sets in $Q_{l, 2 \varepsilon}\left(R_{1, j}, R_{2, j}\right)$ for each $l$. Since $A(z)$ is large in $Q_{l, 2 \varepsilon}\left(R_{1, j}, R_{2, j}\right)$, we deduce that $h_{i}^{\prime} / A^{1 / k}$ must behave asymptotically like $O\left(\frac{z^{M_{2}}}{A^{1 / k}}\right)+d_{i, j}$ at most where $d_{i, j}^{k}+1=0$ for $i=1, \ldots, k$ for some $M_{2}>0$ and $j \geq j_{2}(\varepsilon)$. Therefore,

$$
\left|h_{i}^{\prime}-d_{i, j} A^{1 / k}(z)\right| \leq|z|^{M_{2}+1} \quad \text { for } j \geq j_{2}(\varepsilon), \quad z \in Q_{l, 2 \varepsilon}\left(R_{1, j}, R_{2, j}\right) .
$$

By the triangle inequality,

$$
\left|\sum_{i=1}^{k} h_{i}^{\prime}-\sum_{i=1}^{k} d_{i, j} A^{1 / k}\right| \leq \sum_{i=1}^{k}\left|h_{i}^{\prime}-d_{i, j} A^{1 / k}\right| \leq|z|^{M_{3}}, \quad z \in Q_{l, 2 \varepsilon, H}\left(R_{1, j}, R_{2, j}\right),
$$

holds for some $M_{3}>0$. But $\sum_{i=1}^{k} h_{i}^{\prime}$ must be a polynomial since $E$ has finite order. This implies $\sum_{i=1}^{k} d_{i, j}=0$ for all $j \geq j_{2}(\varepsilon)$.

We may choose any convenient $\zeta_{j} \in Q_{l, 2 \varepsilon, H}\left(R_{1, j}, R_{2, j}\right)$ and this defines, with a suitable branch of $A^{1 / k}$,

$$
G_{i, j}(z)=\int_{\zeta_{j}}^{z} d_{i, j} A(t)^{1 / k} d t
$$


Cauchy's estimate of (8.6) yields

$$
\left|h_{i}^{(m)}\left(r e^{i \theta}\right)-G_{i, j}^{(m)}\left(r e^{i \theta}\right)\right| \leq|z|^{M_{4}}
$$

for $m=1, \ldots, k$ in $Q_{l, 2 \varepsilon, H}\left(R_{1, j}, R_{2, j}\right)$, provided $R_{2, j}-R_{1, j}$ is suitably chosen and $j \geq j_{3}(\varepsilon)$ say (this is possible because $H$ is a locally finite family of discs and there are no member of $H$ in $Q_{l, 2 \varepsilon, H}\left(R_{1, j}, R_{2, j}\right)$ and the path of integration is chosen to be at least a distance $1 / L$ from any exceptional set and the boundary of $\left.Q_{l, 2 \varepsilon, H}\left(R_{1, j}, R_{2, j}\right)\right)$. As in [6], we define new representations by

$$
f_{i}=W_{i, j} e^{G_{i, j}}, \quad i=1, \ldots, k \text {. }
$$

From (8.4) and the representation $f_{i}=\pi_{i} e^{h_{i}}$ we deduce, for some $M_{5}>0$,

$$
\left|\frac{W_{i, j}^{(m)}}{W_{i, j}}\right| \leq|z|^{M_{5}}, \quad m=1, \ldots, k \text { for } z \in Q_{l, 2 \varepsilon, H}\left(R_{1, j}, R_{2, j}\right) \text { and } j \geq j_{3}(\varepsilon) \text {. }
$$

We now substitute this new representation in (4.12) to obtain

$$
\left(G_{i, j}^{\prime}\right)^{k-1}\left(k \frac{W_{i, j}^{\prime}}{W_{i, j}}+\frac{k(k-1)}{2} \frac{G_{i, j}^{\prime \prime}}{G_{i, j}^{\prime}}\right)+\sum_{\mu=0}^{k-2} D_{\mu}(z)\left(G_{i, j}^{\prime}\right)^{\mu}=0
$$

where $D_{\mu}$ is a polynomial in $W_{i, j}^{\prime} / W_{i, j}, G_{i, j}^{\prime \prime} / G_{i, j}^{\prime}$ and their derivatives with constant coefficients. But $A(z)$ is large, so we deduce from (8.8) that

$$
\frac{W_{i, j}^{\prime}}{W_{i, j}}+\frac{k-1}{2} \frac{G_{i, j}^{\prime \prime}}{G_{i, j}^{\prime}}=O\left(r^{-2}\right) \quad \text { for } j \geq j_{4}(\varepsilon)
$$

Differentiating $\log E$, and making use of (8.9) yields

$$
\begin{aligned}
\frac{E^{\prime}}{E} & =\sum_{i=1}^{k} \frac{f_{i}^{\prime}}{f_{i}}=\sum_{i=1}^{k}\left(\frac{W_{i, j}^{\prime}}{W_{i, j}}+G_{i, j}^{\prime}\right)=\sum_{i=1}^{k} \frac{W_{i, j}^{\prime}}{W_{i, j}}+\sum_{i=1}^{k} d_{i, j} A(z)^{1 / k} \\
& =\sum_{i=1}^{k} \frac{W_{i, j}^{\prime}}{W_{i, j}}=-\left(\frac{k-1}{2}\right) \frac{1}{k} \frac{A^{\prime}}{A}+O\left(r^{-2}\right), \quad j \geq j_{4}(\varepsilon) .
\end{aligned}
$$

We deduce

$$
\log \left(E A^{\left(\frac{k-1}{2}\right) \frac{1}{k}}\right)=O(1), \quad j \geq j_{5}(\varepsilon)
$$

and this implies

$$
E \rightarrow 0 \text { in } Q_{l, 2 \varepsilon, H}\left(R_{1, j}, R_{2, j}\right) \text { as } j \rightarrow \infty \text {. }
$$


Thus we deduce $I_{1}=0$ for $r \in Q_{l, 2 \varepsilon, H}\left(R_{1, j}, R_{2, j}\right)$ as $j \geq j_{5}(\varepsilon)$.

Subcase II: $A(z)$ satisfies (ii) of Theorem 4 in $Q_{l, 2 \varepsilon, H}\left(r_{1, j}, r_{2, j}\right)$. By Lemma 9, there exists a path $\Lambda$ such that the circular arcs $\left(\Omega_{j}\right)$ have the same radii as those chosen in Subcase I. By the remark at the end of the proof of Lemma 9 in $\S 7$ we deduce

$$
|E|=\left|f_{1} \cdots f_{k}\right| \leq\left(\gamma^{4 s+2} r^{(2 s+1)(k-1)}\right)^{k} \leq \gamma^{(4 j+2) k} r^{(2 j+2) k(k-1)}, \quad j \geq j_{6}(\varepsilon) .
$$

Therefore

$$
\log |E|=O(4 k j+2 k(k-1)(j+1) \log r),
$$

and so by the hypothesis of $j$ in $\S 4$ (which states that $\log r \sim c j$ ),

$$
\log |E|=O\left((\log r)^{2}\right), \quad r e^{i \theta} \in Q_{l, 2 \varepsilon, H}\left(R_{1, j}, R_{2, j}\right) .
$$

Thus

$$
I_{1}=O\left((\log r)^{2}\right), \quad r e^{i \theta} \in Q_{l, 2 \varepsilon, H}\left(R_{1, j}, R_{2, j}\right) \quad \text { for } j \geq j_{6}(\varepsilon) .
$$

Subcase III: $A(z)$ satisfies (iii) of Theorem 4. As in Subcase II, we have a common $r \in Q_{l, 2 \varepsilon, H}\left(R_{1, j}, R_{2, j}\right)$ so that $|z|=r$ and $z \in \Omega_{j}$. Lemma 8 yields an estimate of $f$ on $\Omega_{j}$

$$
\left|f\left(r e^{i \theta}\right)\right| \leq A_{2}^{j} j^{k j} \exp (\eta(r)(1+j)),
$$

for $j \geq j_{6}(\varepsilon)$. Hence Lemma 8 and the remark in $\S 6$ together imply

$$
\log |E|=O(j+k j \log r+\eta(r)(1+j)) ;
$$

thus

$$
\begin{gathered}
I_{1}=O\left(\eta(r) \log r+k(\log r)^{2} \log \log r\right), \quad r e^{i \theta} \in Q_{l, 2 \varepsilon, H}\left(R_{1, j}, R_{2, j}\right) \\
\text { for } j \geq j_{7}(\varepsilon) .
\end{gathered}
$$

Part B. In order to apply Lemma 2, we note that $\overline{\log } \overline{\operatorname{dens}}\left(H_{P}\right)=0$. We may therefore choose an (exceptional) set of $r$ with upper logarithmic density $\xi<1 / 6$ such that $\xi+0=\xi<1$. We now take the complement of the union of the above two sets; this union has a lower logarithmic density $1-\xi \geq 5 / 6$. Without loss of generality, we may assume that the choice of $R_{1, j}$ and $R_{2, j}$ is in the complement of this set. Hence (see also [8]) for $r$ belonging to this set, we have

$$
\begin{aligned}
\log _{+}^{+}\left|E\left(r e^{i \theta}\right)\right| & \leq \log ^{+}\left|E\left(r e^{i\left(\theta_{l+1}-2 \varepsilon\right)}\right)\right|+\int_{\theta_{l+1}-2 \varepsilon}^{\theta} r\left|\frac{E^{\prime}}{E}\left(r e^{i t}\right)\right| d t \\
& \leq \log ^{+}\left|E\left(r e^{i\left(\theta_{l+1}-2 \varepsilon\right)}\right)\right|+\int_{P_{l+1,2 \varepsilon}} r\left|\frac{E^{\prime}}{E}\left(r e^{i t}\right)\right| d t
\end{aligned}
$$




$$
\leq \log ^{+}\left|E\left(r e^{i\left(\theta_{l+1}-2 \varepsilon\right)}\right)\right|+K(\sigma(E), \xi)\left(2 \varepsilon \log \frac{1}{2 \varepsilon}\right) T(r, E),
$$

where $K(\sigma(E), \xi)$ is a constant depending only on $\sigma(E)$ and $\xi$. Note that the estimate for $\log ^{+}\left|E\left(r e^{i\left(\theta_{l+1}-2 \varepsilon\right)}\right)\right|$ is either given by (8.11) or (8.12) depending on whether $A(z)$ satisfies either (i) or (ii) or (iii) of the hypotheses. Hence

$$
\begin{gathered}
I_{2}=O\left(\eta(r) \log r+2 \varepsilon \log \frac{1}{2 \varepsilon} T(r, E)+(\log r)^{2}\right), \quad r \in\left(R_{1, j}, R_{2, j}\right) \\
\text { for } j \geq j_{7}(\varepsilon)
\end{gathered}
$$

so that (8.13) holds. We take a common $r \in\left(R_{1, j}, R_{2, j}\right)$ so that (8.13) holds, and combine the estimates (8.10-8.12), if any, of Part A and (8.14) of Part B. Then we obtain for all $j \geq j_{8}(\varepsilon)$,

$$
T(r, E)=O\left(\eta(r) \log r+2 \varepsilon \log \frac{1}{2 \varepsilon} T(r, E)+(\log r)^{2}\right),
$$

$r e^{i \theta} \in Q_{l, 2 \varepsilon, H}\left(R_{1, j}, R_{2, j}\right),(8.13)$ holds for $j \geq j_{8}(\varepsilon)$. Since $\varepsilon>0$ is arbitrary and the above argument always applies, we obtain

$$
T(r, E)=O\left(\eta(r) \log r+(\log r)^{2}\right)=O\left(r^{\frac{n+k}{k}} \log r+(\log r)^{2}\right)
$$

on an increasing sequence of $r$ in $\left(R_{1, j}, R_{2, j}\right), j \geq j_{8}(\varepsilon)$. Since the choice of $R_{1, j}$ and $R_{2, j}$ such that $r_{1, j}<R_{1, j}<R_{2, j}<r_{2, j}$ is arbitrary, we conclude that (8.14) remains true for all $r$ except on a set of lower logarithmic density $1-\xi \geq 5 / 6$. Lemma 1 now implies that

$$
T(r, E)=O\left(r^{\frac{n+k}{k}\left(1+2 \xi+\varepsilon_{1}\right)} \log r+(\log r)^{2}\right), \text { for all } r>r_{1}=r_{1}\left(\varepsilon_{1}\right) .
$$

It follows that

$$
\limsup _{r \rightarrow \infty} \frac{\log T(r, E)}{\log r} \leq \frac{n+k}{k}\left(2 \xi+\varepsilon_{1}\right) .
$$

First, let $\varepsilon_{1} \rightarrow 0$, and since $\xi>0$ is arbitrary, we may then let $\xi \rightarrow 0$. Hence $\sigma(E) \leq \frac{n+k}{k}<\sigma(A)$, a contradiction as observed in the beginning of this proof.

\section{Proof of Theorems 2 and 3}

In order to prove Theorems 2 and 3 , we require some knowledge about the growth properties of entire functions satisfying $\delta(0, A)=1$. Although these properties were already contained in $[8$, Lemma 1$]$ and [10], we state them below for the sake of completeness. 
In what follows we shall always assume that $A(z)$ is a transcendental entire function of finite order satisfying $\delta(0, A)=1$. Let us denote $\left\{b_{n}\right\}$ the sequence of zeros of $A(z)$ with the usual convention that they are ordered with increasing moduli (to infinity). We define

$$
c(r)=: \alpha_{0}+\frac{1}{p} \sum_{\left|b_{\nu}\right| \leq r} \frac{1}{b_{\nu}^{p}}
$$

where $\alpha_{0}$ depends only on $A(z)$.

Lemma 10 [10]. Let $c(r)$ be defined as in (9.1). Then the order $A$ is an integer $p$, say. Let $0<\varepsilon<1,0<\delta<1 / e$ and $1<\sigma \leq 36$ be any given numbers, then the following hold.

(i) Let $\alpha=e^{1 /(1+p)}$ and $c_{j}=c\left(r_{j}\right)$ where $j$ is an integer. Then there exists a $j_{a}(\varepsilon)$ such that for all $j \geq j_{a}(\varepsilon)$,

$$
|\log | A(z)\left|-\operatorname{Re}\left(c_{j} z^{p}\right)\right|<4 \varepsilon\left|c_{j}\right| r^{p}, \quad z \in \Gamma_{j}-E_{j},
$$

where

$$
\Gamma_{j}:=\left\{z=r e^{i \theta}: \alpha^{j} \leq r \leq \alpha^{j+3 / 2}\right\}
$$

and $E_{j}$ is a collection of a finite number of discs whose sum of radii is $4 e \delta \alpha^{j+3 / 2}$.

(ii) There exists an $r_{0}$ such that for all $r \geq r_{0}$,

$$
|c(\sigma r)-c(r)|<\varepsilon|c(r)| \text {. }
$$

Let $r_{1, j}:=\alpha^{j}$ and $r_{2, j}:=\alpha^{j+3 / 2}$ and $H:=\bigcup_{j} E_{j}$. Suppose further that $z=r e^{i \theta}$, and $c_{j}=\left|c_{j}\right| e^{i \omega_{j}}$ and $\theta_{1, j}, \ldots, \theta_{2 p, j}$ are the $2 p$ zeros of $\operatorname{Re}\left(c_{j} z^{p}\right)=$ $\left|c_{j}\right| r^{p} \cos \left(p \theta+\omega_{j}\right)$. Then the sets (4.6), (4.7) and (4.8) with $p$ replaced by $2 p$ as defined in $\S 4$ and with $H:=\bigcup_{j} E_{j}$ are such that

$$
\Gamma_{j}=\bigcup_{l=1}^{2 p}\left\{Q_{l, \varepsilon}\left(r_{1, j}, r_{2, j}\right) \cup P_{l, \varepsilon}\left(r_{1, j}, r_{2, j}\right)\right\}
$$

and

$$
\Gamma_{j}-E_{j}=\bigcup_{l=1}^{2 p}\left\{Q_{l, \varepsilon, E_{j}}\left(r_{1, j}, r_{2, j}\right) \cup P_{l, \varepsilon}\left(r_{1, j}, r_{2, j}\right)\right\} .
$$

Here we note that the angular measure of each $Q_{l, \varepsilon}$ is the same.

Since $\alpha=e^{\frac{1}{1+p}}$, where $p \geq 1$ is an integer, it certainly satisfies $0<\alpha \leq 36$, so it follows from (9.4) that $\left|c\left(r_{j+1}\right)-c\left(r_{j}\right)\right|<\varepsilon c\left(r_{j}\right)$ and hence

$$
\left|\omega_{j+1}-\omega_{j}\right| \leq \arcsin \left(\frac{\varepsilon\left|c_{j}\right|}{\left|c_{j}\right|}\right)=\arcsin (\varepsilon) \leq \varepsilon
$$


and so (4.5) is satisfied. It follows that $\bigcup_{j} Q_{l, \varepsilon, H}\left(r_{1, j}, r_{2, j}\right)$ and $\bigcup_{j} P_{l, \varepsilon}\left(r_{1, j}, r_{2, j}\right)$ are connected sets.

Lemma 11 ([8, Lemma 1] and [10, p. 282]). Suppose that $A(z)$ is as defined above, then, given any $\varepsilon>0$ and $0<\delta<1$, there exists $j_{b}(\varepsilon)$ and we have, after a suitable renumbering of $l$ below,

$$
\log |A(z)|>\text { const. } T(r, A) \quad \text { uniformly in } \bigcup_{j \geq j_{b}}^{\infty} Q_{l, \varepsilon, E_{j}}\left(r_{1, j}, r_{2, j}\right)
$$

for $i=1,3, \ldots, 2 p-1$, and

$$
\log |A(z)|<- \text { const. } T(r, A) \quad \text { uniformly in } \bigcup_{j \geq j_{b}}^{\infty} Q_{l, \varepsilon, E_{j}}\left(r_{1, j}, r_{2, j}\right)
$$

for $l=2,4, \ldots, 2 p$.

Lemma 12 ([8], Lemma 2). The collection of the exceptional sets $H$ described in Lemma 10 has upper logarithmic density zero.

Proof of Theorem 2. Assume that $\varepsilon>0$ and $\delta>0$ are given as in the hypotheses. It now follows from Lemma 10 that the order of $\sigma(A)$ is an integer $p$, say. With the notations in (9.5) and (9.6), we have the chains (9.7) and (9.8) for different $l$. As for the exceptional sets $E_{j}$, it follows from (9.3) that the set $E_{j}$ has angular measure $4 e \delta \alpha^{j+3 / 2} / \alpha^{j}=4 e \delta \alpha^{3 / 2}$ at most. Since $\delta$ is arbitrary, we may assume that $\delta$ is so small that its angular measure is $<\Delta$ of Theorem 4 . By Lemma 12 , the collection of the sets $H:=\bigcup E_{j}$ has upper logarithmic density zero as required in Theorem 4 .

Case (i). $\quad P(z)$ is identitically zero. It follows from (9.7) that for $l=1,3, \ldots$, $2 p-1$ the growth of $A(z)$ satisfies (4.9) of Theorem 4; while (9.8) implies that the growth of $A(z)$ satisfies (4.10) for $l=2,4, \ldots, 2 p$. The conclusion now follows from an application of Theorem 4 with the two possible growths of $A(z)$ (i.e., (4.9) and (4.10)) only.

Case (ii). Suppose $P(z) \not \equiv 0$ and $\operatorname{deg} P=n, n+k<k \sigma(A)$. It is clear that for $l=1,3, \ldots, 2 p-1$, we have (9.7) again and so (4.9) is satisfied. While for $l=2,4, \ldots, 2 p$, we have (9.8) and hence $|A(z)+P(z)|=O\left(r^{n}\right)$ satisfies (4.11). The conclusion again follows from Theorem 4.

We remark that when $P \equiv 0$, then the inequality $n+k<k \sigma(A)$ implies $\sigma(A) \geq 2$ in case (ii) and hence case (i) has an independent interest as it includes the case $\sigma(A)=1$.

Proof of Theorem 3. It follows from the well-known inequality $\sum \delta(a, A) \leq$ $\delta\left(0, A^{\prime}\right)$ that $\delta\left(0, A^{\prime}\right)=1$. Thus we may apply Lemmas 10,11 and 12 to the function 
$A^{\prime}(z)$ instead of $A(z)$. We note that the order of $A^{\prime}(z)$ and $A(z)$ are the same, and are both equal to $p$, say. Let $Q_{l, \varepsilon, E_{j}^{\prime}}^{\prime}\left(r_{1, j}, r_{2, j}\right)$ denote the corresponding region for $A^{\prime}(z)$ as that for $A(z)$. The notations that we use for $A^{\prime}$ are in parallel to those of $A(z)$ in Theorem 2. Given $\varepsilon>0$ and $\delta>0$ as allowed by Lemmas 10 and 11, we have the regions $Q_{l, \varepsilon, E_{j}^{\prime}}^{\prime}\left(r_{1, j}, r_{2, j}\right)$ in which $A^{\prime}$ has a growth rate similar to (9.7) except with the function $T\left(r, A^{\prime}\right)$ instead of $T(r, A)$. The exceptional set is denoted by $H^{\prime}=\bigcup E_{j}^{\prime}$, while $r_{1, j}$ and $r_{2, j}$ are the same as those for $A(z)$ in Theorem 2 .

By Proposition 5.12 of [14], we deduce that $\left|A^{\prime}(z)\right| \leq r^{m}|A(z)|$ except for an $R$-set. However, any $R$-set must have finite linear measure and hence zero upper logarithmic density. So it follows from the inequality (9.7), which is now satisfied by $A^{\prime}(z)$, that $A(z)$ must also grow at a comparable rate as $A^{\prime}(z)$ in $\bigcup_{j} Q_{l, \varepsilon, H^{\prime}}^{\prime}\left(r_{1, j}, r_{2, j}\right)$ and outside an $R$-set. We may incorporate the $R$-set into $H^{\prime}$ for our consideration henceforth. Now the union of the $R$-set and $H^{\prime}$ also has zero logarithmic density. Hence we see that $A(z)$ satisfies (4.9) of Theorem 4 in $\bigcup_{j} Q_{l, \varepsilon, H^{\prime}}^{\prime}\left(r_{1, j}, r_{2, j}\right)$, for $l=1,3, \ldots, 2 p-1$, say. In the remaining regions $A^{\prime}(z)$ has the equivalent form of $(9.8)$ for $l=2,4, \ldots, 2 p$. We now cite a further result from [10, Theorem 3] which asserts that there exists $p$ asymptotic paths and asymptotic values (they are finite but not necessary distinct) of $A(z)$, with each path lying in one of $\bigcup_{j} Q_{l, \varepsilon, H^{\prime}}^{\prime}\left(r_{1, j}, r_{2, j}\right), l=2,4, \ldots, 2 p$, so that along any one of which $A^{\prime}(z)$ tends to zero in the same manner as (9.8) with $A$ replaced by $A^{\prime}(z)$. Let $z_{l, j}$ be a point on one of the asymptotic paths lying in $Q_{l, \varepsilon, H^{\prime}}^{\prime}\left(r_{1, j}, r_{2, j}\right), l=2,4, \ldots, 2 p$, and we set, for any $z \in Q_{l, \varepsilon, H^{\prime}}^{\prime}\left(r_{1, j}, r_{2, j}\right)$,

$$
A(z)=\int_{z_{l, j}}^{z} A^{\prime}(\zeta) d \zeta+A\left(z_{l, j}\right)
$$

where the path of integration can be taken in $Q_{l, \varepsilon, H^{\prime}}^{\prime}\left(r_{1, j}, r_{2, j}\right)$ so that its length is no longer than a constant times $\alpha^{j}$ (since $\left.r_{2, j}-r_{1, j}=O\left(\alpha^{j}\right)\right)$.

Since $A\left(z_{l, j}\right)$ tends to an asymptotic value, and that

$$
\left|\int_{z_{l, j}}^{z} A^{\prime}(\zeta) d \zeta\right|=O\left(\alpha^{j} e^{-\pi / 20 T\left(\alpha^{j}, A^{\prime}\right)}\right)=o(1)
$$

as $j \rightarrow+\infty([10$, p. 287]), it follows from (9.9), provided that we assume $j$ is chosen sufficiently large, that $A(z)$ is bounded by twice the maximum modulus of the largest finite asymptotic values. Hence $A(z)$ is bounded in each $Q_{l, \varepsilon, H^{\prime}}^{\prime}\left(r_{1, j}, r_{2, j}\right)$ provided $j$ is large enough. Thus $A(z)+P(z)=O\left(r^{n}\right)$ in $\bigcup_{j} Q_{l, \varepsilon, H^{\prime}}^{\prime}\left(r_{1, j}, r_{2, j}\right)$ and thus (4.11) of Theorem 4 is fulfilled. This completes the proof of Theorem 3.

\section{Remarks and examples}

The following was proved in [9]. 
Theorem G. Let $K \in \mathbb{C}$. Then the equation

$$
f^{\prime \prime \prime}-K f^{\prime}+e^{z} f=0
$$

admits a fundamental set of solutions $f$ with $\lambda(f)<+\infty$ if and only if there exists a non-negative integer $n$ such that $K=(n+1)^{2} / 9$ and that $n$ satisfies certain tridiagonal $(n+1) \times(n+1)$-determinant condition.

It is conjectured in [9] that the $(n+1) \times(n+1)$-determinant condition holds if and only if $n$ is not of the form $3 k+2$ where $k=0,1,2, \ldots$.

Since $\sum \delta\left(a, e^{z}\right)=1=\delta\left(0, e^{z}\right)$, Theorem $\mathrm{G}$ shows that part (ii) of Theorem 2 , and Theorem 3 (when $P \equiv 0$ ) are sharp when $k=3$ in the sense that the same conclusion does not hold if we allow intermediate terms in (4.1), (4.2) and (4.3). In fact we may consider a special case of equation (10.1), namely, the equation $f^{\prime \prime \prime}-\frac{1}{9} f^{\prime}+e^{z} f=0$, which possesses three linearly independent zero-free solutions of the forms $f(z)=\exp \left(c e^{z / 3}-z / 3\right), c^{3}+27=0$. On the other hand, we note that $\sigma\left(e^{z}\right)=1$ and hence the inequality $n+k<k \sigma\left(e^{z}\right)$ just fails in Theorems 2 and 3 when $k=3$. We refer to [9] for further details.

\section{Concluding remarks}

It is evident that we could relax the assumption that $\overline{\log } \overline{\operatorname{dens}}\left(H_{P}\right)=0$ in the proof of Theorem 4. However, this will make the argument unnecessarily complicated. We choose not to pursue this path here.

Bank and Langley, [4], p. 457, showed that every solution $f(z)$ of $f^{(k)}+\left(\Pi e^{P}+\right.$ Q) $f=0$ must have $\lambda(f)=+\infty$, whenever $P$ and $Q$ are polynomials such that $k+\operatorname{deg} Q<k \operatorname{deg} P$ and $\Pi$ is an entire function of order strictly less than the degree of $P$ (plus some further restrictions). Clearly $\delta\left(0, \Pi e^{P}\right)=1$. Hence, Theorem 2 weakens one of their hypotheses; namely, that our result allows any coefficient $A$ with $\delta(0, A)=1$, but at the expense of getting $\max _{i}\left(f_{i}\right)=+\infty$, for any fundamental set of solutions instead of $\lambda(f)=+\infty$ for all non-trivial solutions. In closing we remark that the case when $k+\operatorname{deg} Q>k \operatorname{deg} P$ was treated in [5], with the same conclusion. Hence, we may ask whether our results remain valid under these hypotheses.

Acknowledgement. The authors would like to acknowledge Pengcheng Wu for his valuable advice on Lemma 1.

\section{References}

[ 1 ] S. Bank and I. Laine, On the oscillation theory of $f^{\prime \prime}+A f=0$ where $A$ is entire, Trans. Amer. Math. Soc., 273 (1982), 351-363.

[ 2 ] S. Bank, I. Laine and J. Langley, On the frequency of zeros of solutions of second-order linear differential equations, Resultate Math., 10 (1986), 8-24. 
[ 3 ] S. Bank, I. Laine and J. Langley, Oscillation results for solutions of linear differential equations in the complex domain, Resultate Math., 16 (1989), 3-15.

[4] S. Bank and J. Langley, On the oscillation of solutions of certain linear differential equations in the complex domain, Proc. Edinburgh Math. Soc., (2) 30 (1987), 455-469.

[5] S. Bank and J. Langley, On the zeros of the solutions of the equation $w^{(k)}+\left(R e^{P}+Q\right) w=0$, Kodai Math. J., 13 (1990), 298-309.

[6] S. Bank and J. Langley, Oscillation theory for higher order linear differential equations with entire coefficients, Complex Variables Theory Appl., 16 (1991), 163-175.

[ 7 ] Y.M. Chiang, Schwarzian derivative and second order differential equations, Ph.D. thesis, London, 1991.

[8] Y.M. Chiang, Oscillation results on $y^{\prime \prime}+A y=0$ in the complex domain with transcendental entire coefficients which have extremal deficiencies, Proc. Edinburgh Math. Soc., 38 (1995), 13-34.

[ 9 ] Y.M. Chiang, I. Laine and S. Wang, An oscillation result of a third order linear differential equation with entire periodic coefficients, Complex Variables Theory Appl., 34 (1997), 25-34.

[10] A. Edrei and W.H.J. Fuchs, Valeurs déficientes et valeurs asymptotiques des fonctions méromorphes, Comment. Math. Helv., 33 (1959), 258-295.

[11] W.H.J. Fuchs, Proof of a conjecture of G. Pólya concerning gap series, Illinois J. Math., 7 (1963), 661-667.

[12] W.K. Hayman, Meromorphic functions, Clarendon Press, Oxford, 1964.

[13] H. Herold, Ein Vergleichssatz für komplexe lineare Differentialgleichungen, Math. Z., 126 (1972), 91-94.

[14] I. Laine, Nevanlinna Theory and Complex Differential Equations, W. de Gruyter, Berlin, 1993.

\author{
Y. M. CHIANG \\ Department of MATHEMAtics \\ THE HONG KONG UNIVERSITY \\ of Science And TeChNology \\ Clear Water Bay, Kowloon \\ HONG KONG \\ I. LAINE \\ Department of MAThematics \\ UNIVERSITY OF JOENSUU \\ P.O. Box 111 \\ FIN-80101 JOENSUU \\ FINLAND
}

